ARTICLE

\title{
Directional reorientation of migrating neutrophils is limited by suppression of receptor input signaling at the cell rear through myosin II activity
}

\author{
Amalia Hadjitheodorou (1) ${ }^{1,2}$, George R. R. Bell (10 ${ }^{3}$, Felix Ellett ${ }^{4}$, Shashank Shastry ${ }^{3}$, Daniel Irimia (1) ${ }^{4}$,
} Sean R. Collins ${ }^{3 凶} \&$ Julie A. Theriot (1) ${ }^{2 凶}$

To migrate efficiently to target locations, cells must integrate receptor inputs while maintaining polarity: a distinct front that leads and a rear that follows. Here we investigate what is necessary to overwrite pre-existing front-rear polarity in neutrophil-like HL60 cells migrating inside straight microfluidic channels. Using subcellular optogenetic receptor activation, we show that receptor inputs can reorient weakly polarized cells, but the rear of strongly polarized cells is refractory to new inputs. Transient stimulation reveals a multi-step repolarization process, confirming that cell rear sensitivity to receptor input is the primary determinant of large-scale directional reversal. We demonstrate that the RhoA/ROCK/ myosin II pathway limits the ability of receptor inputs to signal to $\mathrm{Cdc} 42$ and reorient migrating neutrophils. We discover that by tuning the phosphorylation of myosin regulatory light chain we can modulate the activity and localization of myosin II and thus the amenability of the cell rear to 'listen' to receptor inputs and respond to directional reprogramming.

\footnotetext{
${ }^{1}$ Department of Bioengineering, Stanford University, Stanford, CA, USA. ${ }^{2}$ Department of Biology and Howard Hughes Medical Institute, University of Washington, Seattle, WA, USA. ${ }^{3}$ Department of Microbiology and Molecular Genetics, University of California, Davis, Davis, CA, USA. ${ }^{4}$ Department of Surgery, BioMEMS Resource Center, Massachusetts General Hospital, Harvard Medical School, Boston, MA, USA. ${ }^{凶}$ email: srcollins@ucdavis.edu; jtheriot@uw.edu
} 
$\mathrm{N}$ eutrophils, the most abundant circulating leukocytes in humans, comprise the first line of innate immune defense. Their directed migration is mediated by detection of chemoattractants such as N-formyl-methionyl-leucyl-phenylalanine (fMLF) via G protein-coupled receptors (GPCRs), fairly evenly distributed on the cell surface ${ }^{1,2}$. Ligand binding to the receptor activates signaling via $G_{\alpha i}$, leading to cell polarization and directional motility ${ }^{3}$.

An early manifestation of neutrophil polarization is the generation of steep antagonistic gradients of Cdc42 and RhoA activity, formed synchronously towards the cell front and rear, respectively ${ }^{4}$ (Fig. 1a). At the front, Cdc42 and Rac induce actin polymerization, whereas RhoA regulates myosin II contractility at the rear. The front and rear signaling modules are mutually exclusive $^{3}$ and are governed by positive feedback loops for selfamplification and polarity maintenance ${ }^{5-7}$. In addition, tension by the plasma membrane has been demonstrated to act as a longrange inhibitor, mechanically preventing the generation of multiple cell fronts ${ }^{8}$.

Once established, front-rear polarity in migrating neutrophils is thought to be relatively stable, as evidenced by the observation that polarized neutrophils often steer their original front to make a U-turn instead of repolarizing, suggesting that neutrophils are more sensitive to chemoattractants towards their front as compared to their rear $^{1}$. To examine the role of polarity in chemoattractant sensing, researchers have historically challenged neutrophils migrating on two-dimensional (2D) planar substrates using point sources of chemoattractant at different angles with respect to the original direction of migration ${ }^{3,9-11}$. In these experiments, chemoattractant is typically delivered using a micropipette positioned near the cell, resulting in diffusion of the attractant over the entire cellular surface. Thus, it is difficult to decouple whether the rear was intrinsically less responsive to chemoattractant signaling than the front, or whether the greater amplification of signaling inputs at the cell front gave it the advantage. Recent studies have leveraged microfluidic devices, examining the response of neutrophil-like HL60 cells and Dictyostelium cells to flipping the direction of the chemoattractant gradient when cells are confined in one-dimensional (1D) channels and cannot physically perform a U-turn ${ }^{12,13}$

To probe the cellular sensitivity to receptor inputs at the level of signaling, and to determine what is necessary to overwrite front-rear polarity, we used an optogenetic approach to locally activate $G_{\alpha i}$ signaling, independent of fMLF, and drive cells to dynamically repolarize without modifying the environment in which they are embedded. We found that persistent optogenetic receptor activation at the rear of neutrophils migrating in $1 D$ microfluidic channels is sufficient to reorient weakly polarized and slowly migrating cells. However, in more strongly polarized cells, myosin II and RhoA activity limit the ability of the rear to respond, even at the level of signal transmission to Cdc42, creating a cell rear that is refractory to new receptor inputs. We show that by applying pharmacological treatments known to alter the phosphorylation of myosin regulatory light chain (MRLC), we can modulate the activity and localization of myosin II and thus the amenability of the cell rear and the ability of cells to reverse their direction of motion.

\section{Results}

Persistent optogenetic stimulation is sufficient to reverse weakly polarized and slowly migrating cells. We generated a neutrophil-like HL60 cell line stably expressing parapinopsin (a light-sensitive $\mathrm{G}_{\mathrm{ai}}$-family GPCR) and a tdTomato/tdKatushka2 Cdc42 fluorescence resonance energy transfer (FRET) biosensor, spectrally compatible with the reversible parapinopsin stimulation. This system allows direct recording of downstream Cdc42 activity in cells whose migration is guided by parapinopsin 14 . We confined cells to migrate inside 1D straight microfluidic channels in response to spatial serum gradients (Fig. 1a). Using a $407 \mathrm{~nm}$ laser, we locally stimulated cells with $10 \mathrm{~ms}$ light pulses and the spatial precision of about a $1 \mu \mathrm{m}$ diameter spot, using real-time image analysis to automatically and dynamically position the activation spot at the appropriate location (see "Methods"). We found that repeatedly delivering light pulses every $3 \mathrm{~s}$ at the cell rear was sufficient to overwrite the front-rear polarity, drive a chemotaxis-like response at the level of $\mathrm{Cdc} 42$, and ultimately reverse the direction of motion in a subset (about 47\%) of cells (Fig. 1b and Supplementary Movie 1).

We were initially surprised by the observation that just $47 \%$ of cells reversed (Fig. 1c). To better understand why only some cells were able to reverse direction, we first used flow cytometry to measure the mCitrine-tagged parapinopsin receptor (abbreviated as $\mathrm{mCit}$ on Fig. 1a) and found that over $97 \%$ of cells expressed the construct (Supplementary Fig. 1a, b). We next confirmed that at least $70-93 \%$ of cells were responsive to a five-pulse optical stimulation administered at their center (Supplementary Fig. 1c-e and Supplementary Movie 2). These estimates of inherently responsive cells were significantly higher than the $47 \%$ of reversing cells, suggesting that the reversals were suppressed due to some other kind of variation among cells.

We then examined whether there were any measurable prestimulation differences between reversing and non-reversing cells. Reversing cells tended to be weakly polarized (Fig. 1d, e) and slower migrators (Fig. 1f), although there was considerable overlap between the distributions of reversing and non-reversing cells. The primary determinant of reversibility came from the cell rear (Supplementary Fig. 2a-c). Mean front/rear Cdc42 activity ratio appeared to correlate with centroid speed (Fig. 1g). We found no correlation between a cell's position along the channel or a cell's differentiation age and its likeliness to repolarize upon stimulation (Supplementary Fig. 2d, e).

All together, our analysis suggested that pre-existing variations in cell state could be influencing the ability of the cell to respond to new receptor inputs, and that the amenability of a cell to reverse polarity may be tunable by the sensitivity of the cell rear.

In reversing cells, Cdc42 activation at the stimulated rear begins immediately and cells reverse their direction of migration before $\mathrm{Cdc42}$ activity flips polarization. To gain further insight into what may be controlling the rear sensitivity to receptor inputs, we studied the stepwise order of events during optogenetic-driven cell reversals. We quantified the Cdc42 activity at the original rear and front for 78 cells that reversed (Fig. 2a) as well as the derivative of Cdc42 activity at each edge (Fig. 2b). Notably, Cdc42 activity began to increase at the stimulated rear almost immediately, whereas the decrease in Cdc42 activity at the old front had a much slower onset. Most strikingly, the direction of migration flipped $27 \mathrm{~s}$ post stimulation (Fig. 2c), considerably before the two cell edges reached equal levels of Cdc42 activity (Cdc42 cross-point) $51 \mathrm{~s}$ post stimulation. Thus, the cell edge that has more Cdc42 activity is not necessarily the driving front.

Moreover, as the stimulated original rear began to increase local Cdc42 activity, the two cell edges pulled in competing directions, causing the normalized cell length to increase and peak $45 \mathrm{~s}$ after the initiation of stimulation (Fig. 2d), temporally close to the Cdc 42 cross-point. The normalized cell area remained constant during this tug-of-war between the original and emerging front (Fig. 2d). We conclude that the stimulated rear quickly assumed front character and started to move towards the opposite direction, before the signal was transmitted to the 
a

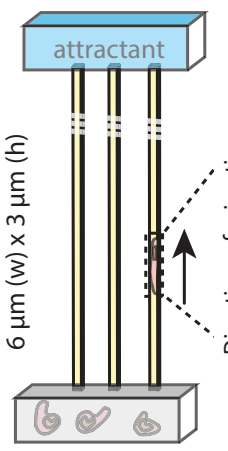

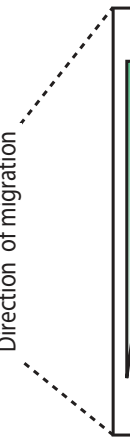
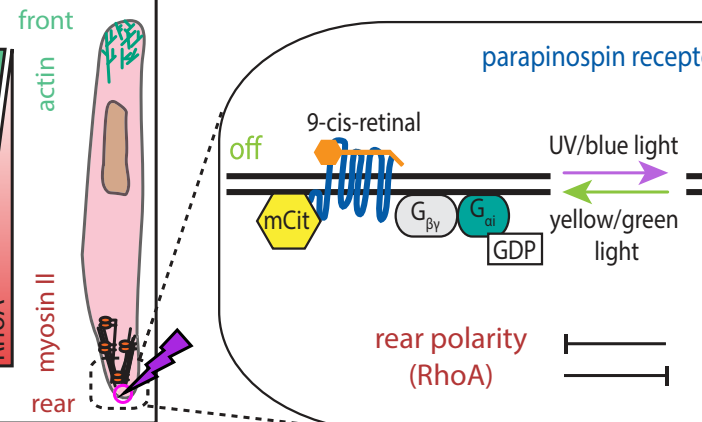

rear polarity

(RhoA)

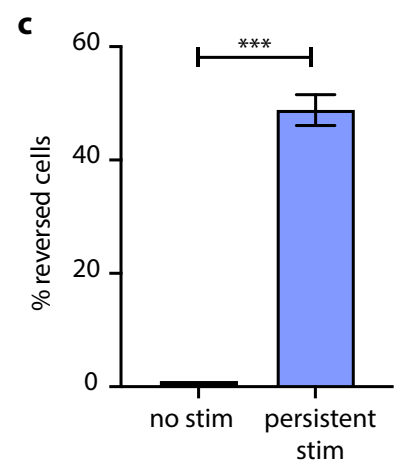

d

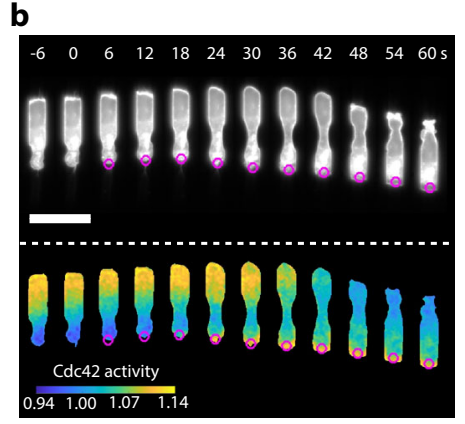

e

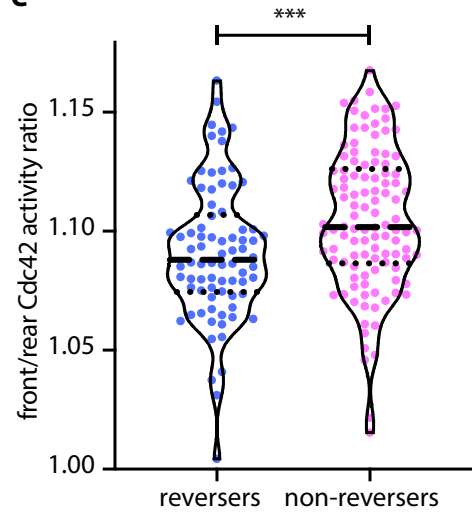

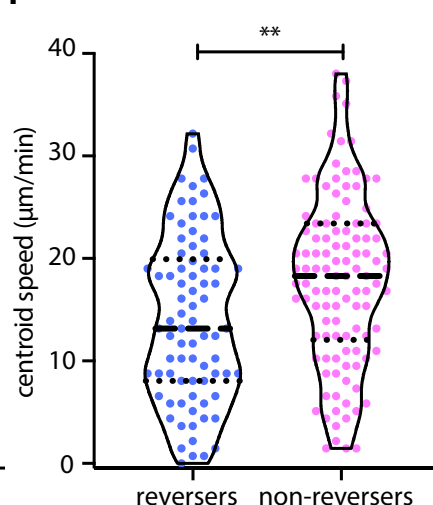

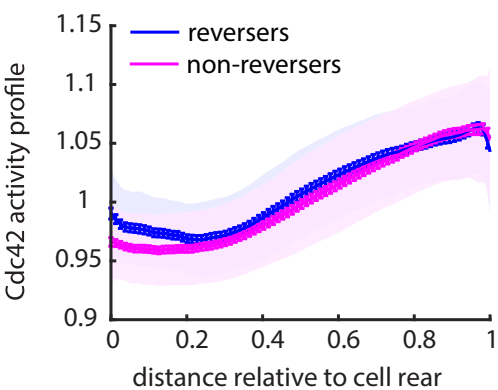

g

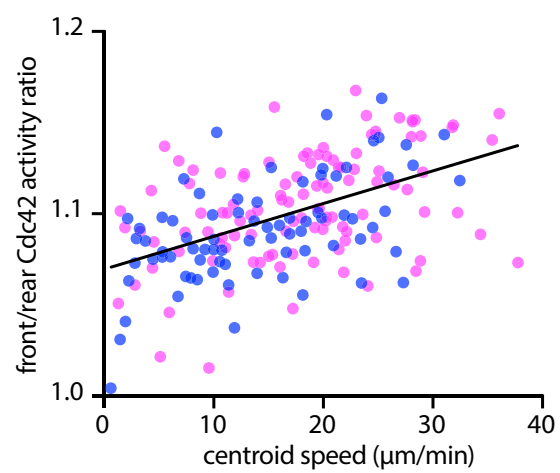

Fig. 1 Persistent optogenetic stimulation is sufficient to reverse weakly polarized and slowly migrating cells. a Schematic representation of the front-rear polarity reversal assay. HL60 cells expressing parapinopsin, an optogenetic receptor, migrate inside microfluidic devices that harbor straight channels. A heat-inactivated serum gradient is used as an attractant. The opsin can be turned on using UV/blue light and off with yellow/green light, offering complete control of the receptor activity. Blue light stimulation (magenta circle/lighting bolt) at the rear of the cell turns on the receptor. The receptor is coupled to the polarity signal transduction network and activation re-enforces the front module. $\mathbf{b}$ Live-cell imaging snapshots of cells expressing parapinopsin and a red/far-red Cdc42 FRET sensor. Cells migrate unperturbed for $60 \mathrm{~s}$ prior to initiation of persistent pulsed stimulation (magenta circles) at their rear. Upper and lower panels show the registered sensors (gray scale) and the computed Cdc42 activity, respectively. Images were captured every $3 \mathrm{~s}$ and subsampled for illustration purposes. Scale bar: $25 \mu \mathrm{m}$. c Box plot of the percentage of cells that reversed with persistent pulsated stimulation ( $n=336$ cells from 36 independent experiments) vs. non-stimulated control ( $n=32$ cells from 3 independent experiments). Error bar represents confidence intervals assuming a binomial distribution around the cumulative mean. Two-sided Fisher's exact test revealed a significant difference between the two conditions ( ${ }^{\star \star *} p<0.001$ ). d Mean Cdc42 activity profiles for $n=78$ reverser (blue) and $n=110$ non-reverser (magenta) cells averaging cell profiles over $15 \mathrm{~s}$ prior to stimulation (lines: means, shaded regions: SD, error bars: mean value $\pm \mathrm{SE}$ ). e, $\mathbf{f}$ Violin plots of mean front/rear Cdc42 activity ratio (e) and of mean centroid speed (f) of $n=78$ reverser and $n=110$ non-reverser cells, averaging over $15 \mathrm{~s}$ prior to stimulation; $p$-values of two-sided Wilcoxon's rank-sum test $\left.{ }^{\star \star \star} p<0.01,{ }^{\star \star \star} p<0.001\right)$. $\mathbf{g}$ Linear regression between calculated mean front/rear Cdc42 activity, shown in $\mathbf{e}$, and mean centroid speed, shown in $\mathbf{f}$, prior to stimulation, for $n=188$ cells, yields Pearson's correlation coefficient $r=0.502, p=2 \times 10^{-13}$. Source data are provided as a Source Data file, including exact $p$-values.

original front. In other words, flipping polarity was not instantaneous, as flipping a switch, but was a multi-step transition.

The above order of events was also evident in single cells (Fig. 2e, f). Phase diagrams of mean centroid speed vs. mean rear/ front Cdc42 activity ratio (Fig. $2 \mathrm{~g}$ ) and of mean rear vs. mean front Cdc42 activity (Fig. 2h) further illustrated the transitional states a cell occupied before flipping its polarity and resuming a new steady state (Fig. 2i). This analysis confirmed that Cdc42 activity at the stimulated rear rose quickly, and only after the Cdc42 activity at the original rear got quite high did the Cdc42 activity at the original front start decreasing. 
a

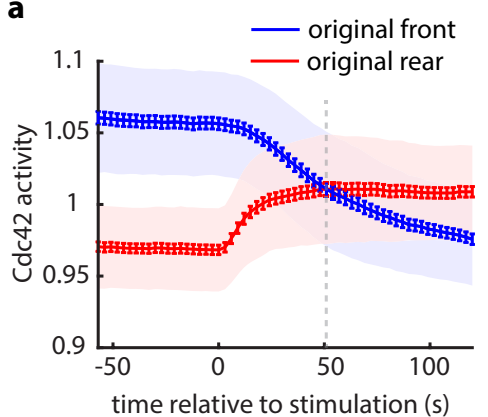

d

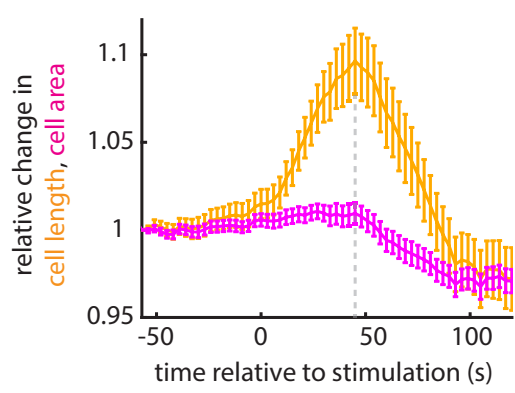

b

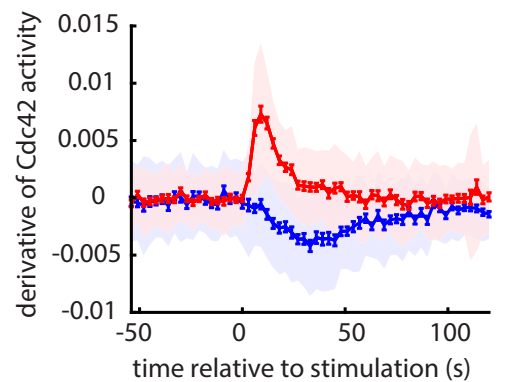

e

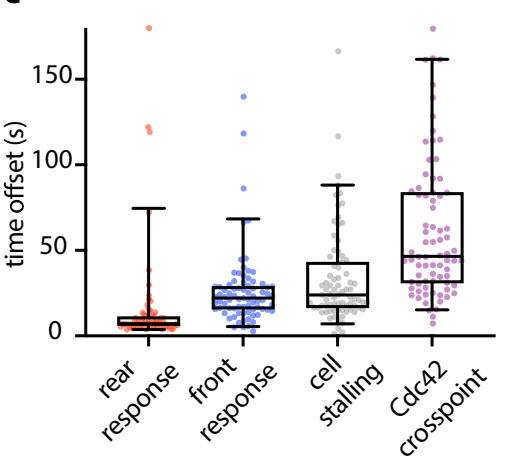

c

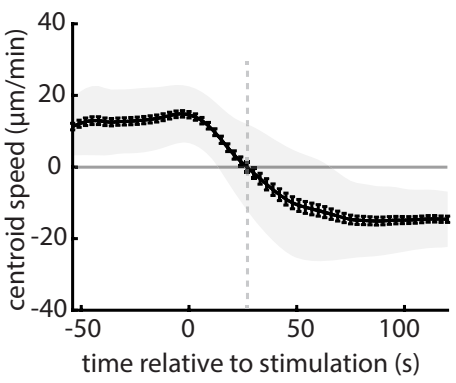

f (1) (2) (3) (4)

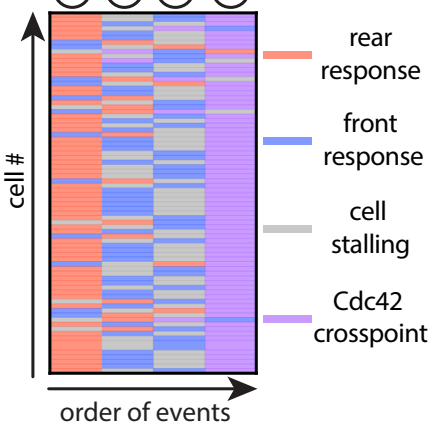

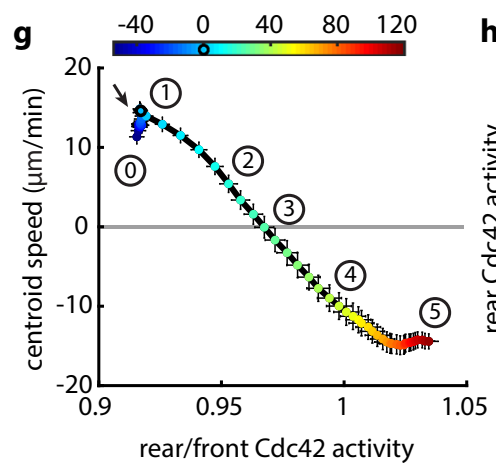

h

i

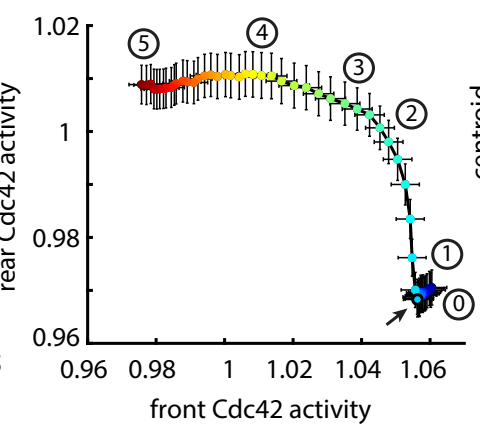

(2) (1) (2) (3) (4) (5)

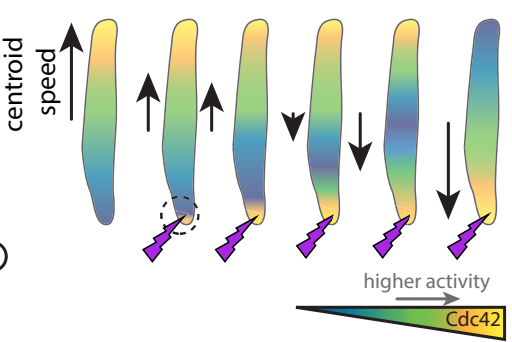

original front responds ( $21 \mathrm{~s})$

(3) cell direction

(4) equal Cdc42 activity
at front and rear $(\sim 51 \mathrm{~s})$

(5)
state

Fig. 2 In reversing cells, Cdc42 activation at the stimulated original rear begins immediately and cells reverse their direction of migration before

Cdc42 activity flips polarization. a, b Mean magnitude (a) and derivative (b) of Cdc42 activity at the original cell front (blue) and rear (red) over time (lines: means, shaded regions: SD, error bars: mean value \pm SE). Data are averages from $n=78$ reversed cells from 36 independent experiments. Dashed vertical line in a shows the Cdc42 cross-point. c Centroid speed of cells that reversed their migratory direction over time (line: mean, shaded region: SD, error bars: mean value $\pm \mathrm{SE}$ ). Data are averages from the same 78 cells shown before. Dashed vertical line indicates when cell stalling (zero speed) occurs. d Relative change in cell length (orange) and cell area (magenta), both normalized to the initial length and area for each of the same 78 cells (lines: means, error bars: mean value \pm SE). Dashed vertical line indicates the time when the relative change in cell length reaches maximum value. Note that the normalized area appeared to decrease post stimulation, overshooting below the starting value. This relative decrease in cell area was associated with a smaller rear after repolarization. Moreover, the $\mathrm{Cdc} 42$ activity at the new emerging front was restricted in a smaller depth as compared to the pre-stimulus steady state (Supplementary Movie 1), suggesting that the repolarized cell adopted a sharper Cdc42 activity profile with a more constricted rear. e Box plots of the time offset for rear response, front response, cell stalling, and Cdc42 cross-point for the same 78 cells that reversed. Box plots extend from the 25th to the 75th percentiles; lines inside box plots represent the medians and whiskers are drawn to the 5th percentile and up to the 95th. $\mathbf{f}$ Heat map of the order of events (columns) across the 78 reversed cells shown before (rows). Circled numbers correspond to the cell states shown in $\mathbf{i}$. $\mathbf{g}$, $\mathbf{h}$ Phase diagrams of mean centroid speed vs. mean rear/front Cdc42 activity $(\mathbf{g})$ and of mean rear vs. mean front Cdc42 activity (h), averaging over the same 78 reversed cells. Colored points correspond to the average cell state at different time points relative to the start of the persistent pulsated stimulation (black circle/arrow). Circled numbers indicate the cell states as summarized in $\mathbf{i}$ and error bars denote mean value \pm SE. $\mathbf{i}$ Cartoon depiction of the average order of events for a cell that reverses. Source data are provided as a Source Data file. 

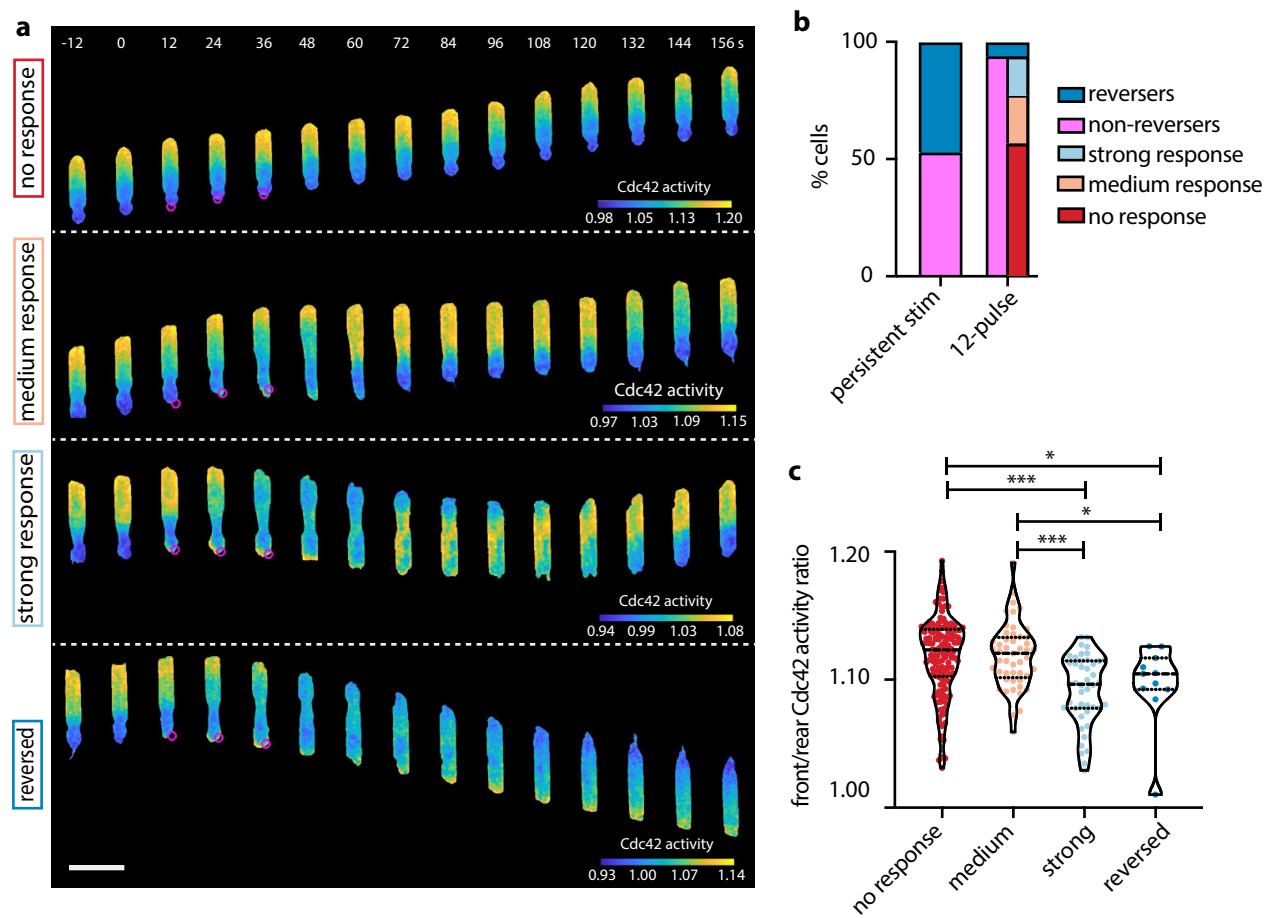

Fig. 3 Transient stimulation reveals a variety of distinct cellular responses. a Representative live-cell imaging snapshots of stimulation experiments with cells expressing parapinopsin and a red/far-red Cdc42 FRET sensor, showing no response (upper panel), medium response, strong response, and a reversed response (lower panel). Cells migrated unperturbed for $60 \mathrm{~s}$ prior to starting a transient 12-pulse stimulation at their cell rear (magenta circles). Images were captured every $3 \mathrm{~s}$ and subsampled for illustration purposes. Scale bar: $25 \mu \mathrm{m}$. $\mathbf{b}$ Stacked bar plot of the percentage of cells that exhibited each cellular response with persistent pulsated stimulation ( $n=336$ cells from 36 independent experiments) and with transient 12-pulse stimulation ( $n=261$ cells from 20 independent experiments). c Violin plot of mean front/rear Cdc42 activity ratio of $n=141$ non-responders, $n=49$ medium responders, $n=44$ strong responders, and $n=11$ reversers, averaging over $15 \mathrm{~s}$ prior to stimulation; $p$-values of two-sided Wilcoxon's rank-sum test $\left({ }^{\star} p<0.05,{ }^{\star \star \star} p<0.001\right.$, pairs not shown have $\left.p>0.05\right)$. Source data are provided as a Source Data file, including exact $p$-values.

Transient stimulation reveals a variety of distinct cellular responses. Surprised by the observation that cells reversed their direction of migration before Cdc42 activity flipped polarization, we asked what would happen if we stopped the stimulation in between these two key events, namely after the cell stalling at $27 \mathrm{~s}$ and before the Cdc42 cross-point at $51 \mathrm{~s}$. We found that transient 12-pulse stimulation resulted in four distinct classes of observed cellular responses that manifested differently at the level of Cdc42 activity with distinct migration patterns (Fig. 3a and Supplementary Movie 3). Fifty-six percent of cells showed no measurable response, maintaining their original speed and showing no or minimal rise $(<2 \%)$ in Cdc42 activity at their rear despite the stimulation. Twenty-one percent of cells showed some increase of Cdc42 activity at their rear (>2\%) but no engagement of the front, which appeared strongly polarized and unyielding, resulting in only cell rear elongation. We termed this class "medium response". Seven percent of cells exhibited a "strong response," transiently flipping their direction of motion, but with Cdc42 polarity and migration reverting back to their original polarization post stimulation. The remaining $6 \%$ of cells reversed. Transient stimulation was significantly less effective in overwriting polarity and reorienting cells as compared to persistent stimulation (6\% instead of $47 \%$ reversing cells, respectively; Fig. 3b). Notably, the fraction of non-responding cells in the 12pulse assay was comparable to the fraction of cells that did not reverse upon persistent stimulation (56\% and 53\%, respectively; Fig. $3 b$ ). This suggests that cells forming the medium and strong response classes may represent cells with pre-existing conditions that permitted responsiveness and repolarization, but required greater stimulus inputs to completely reverse direction.
We asked whether we could identify any pre-stimulation variations among cells that exhibited these distinct responses. We found that strong responding and reversing cells were slower migrators with weaker polarization than cells that showed no or medium response (Supplementary Fig. 3a and Fig. 3c). Nonresponding cells had the strongest rears (i.e., lowest rear-localized Cdc42 activity) among all classes (Supplementary Fig. 3b, c). Lastly, front Cdc42 activity was higher in medium compared to strong responding cells (Supplementary Fig. 3d), whose front eventually yielded to the original rear and transiently repolarized.

One possible explanation for why strong responding cells regained their original polarity orientation could be that the preexisting serum gradient might have flipped back their polarity axis after we switched off the optogenetic stimulus. We therefore examined what would happen if we performed similar experiments for cells moving through channels in a uniform serum environment instead of a gradient, but found no significant differences (Supplementary Fig. 3e), indicating that detection of the pre-existing serum gradient cannot explain why strong responding cells reorient back toward their original direction of motion after optogenetic stimulation ceases. In addition, no significant differences were found when comparing prestimulation polarization states of cells migrating in a uniform serum environment vs. a gradient (Supplementary Fig. 3f-j).

A strong cell rear is refractory to receptor inputs. We quantified the average centroid speed traces for the four different classes, namely no response, medium response, strong response, and reversed (left to right, Fig. 4a), as well as the magnitude and 
a
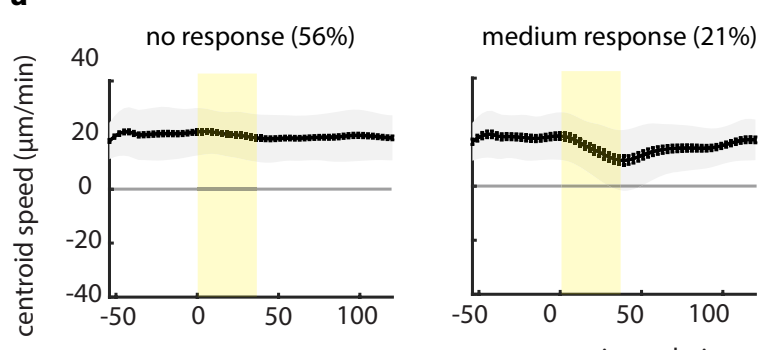

strong response (17\%)
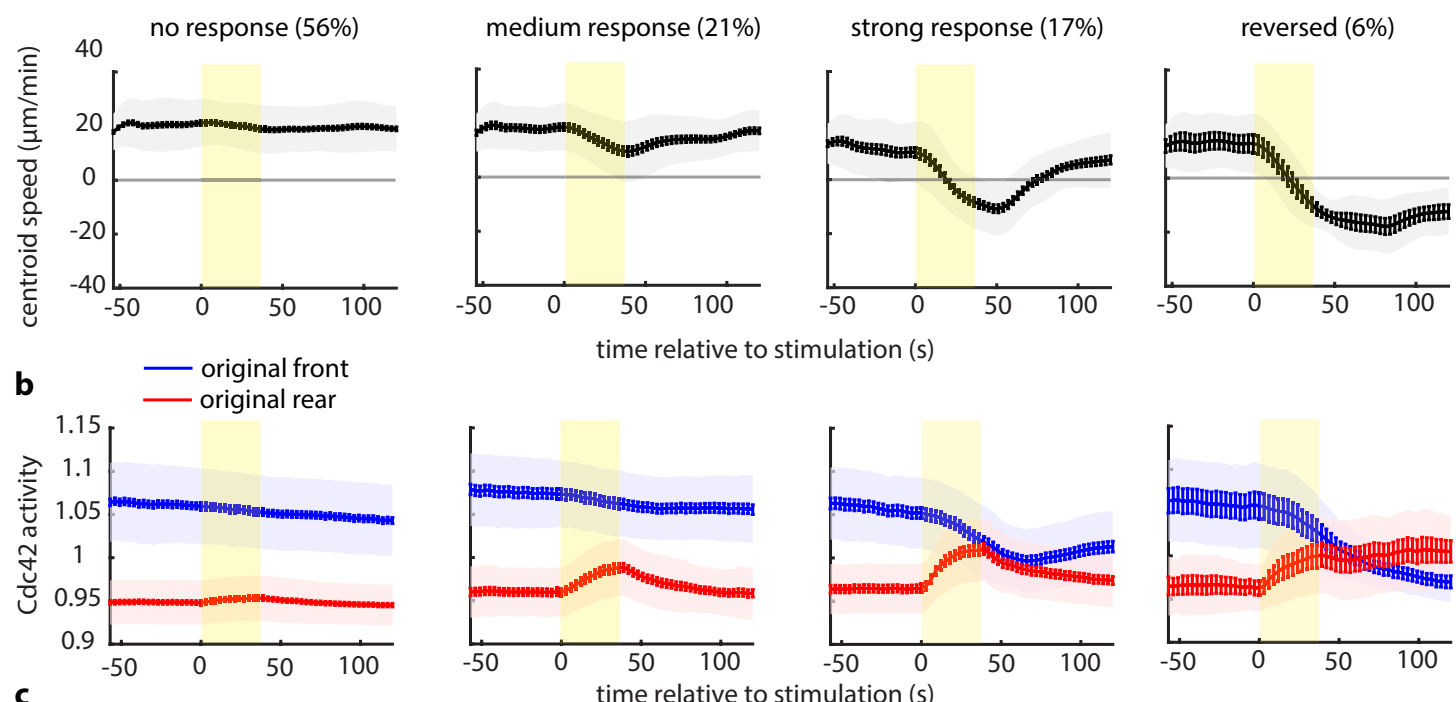

time relative to stimulation (s)
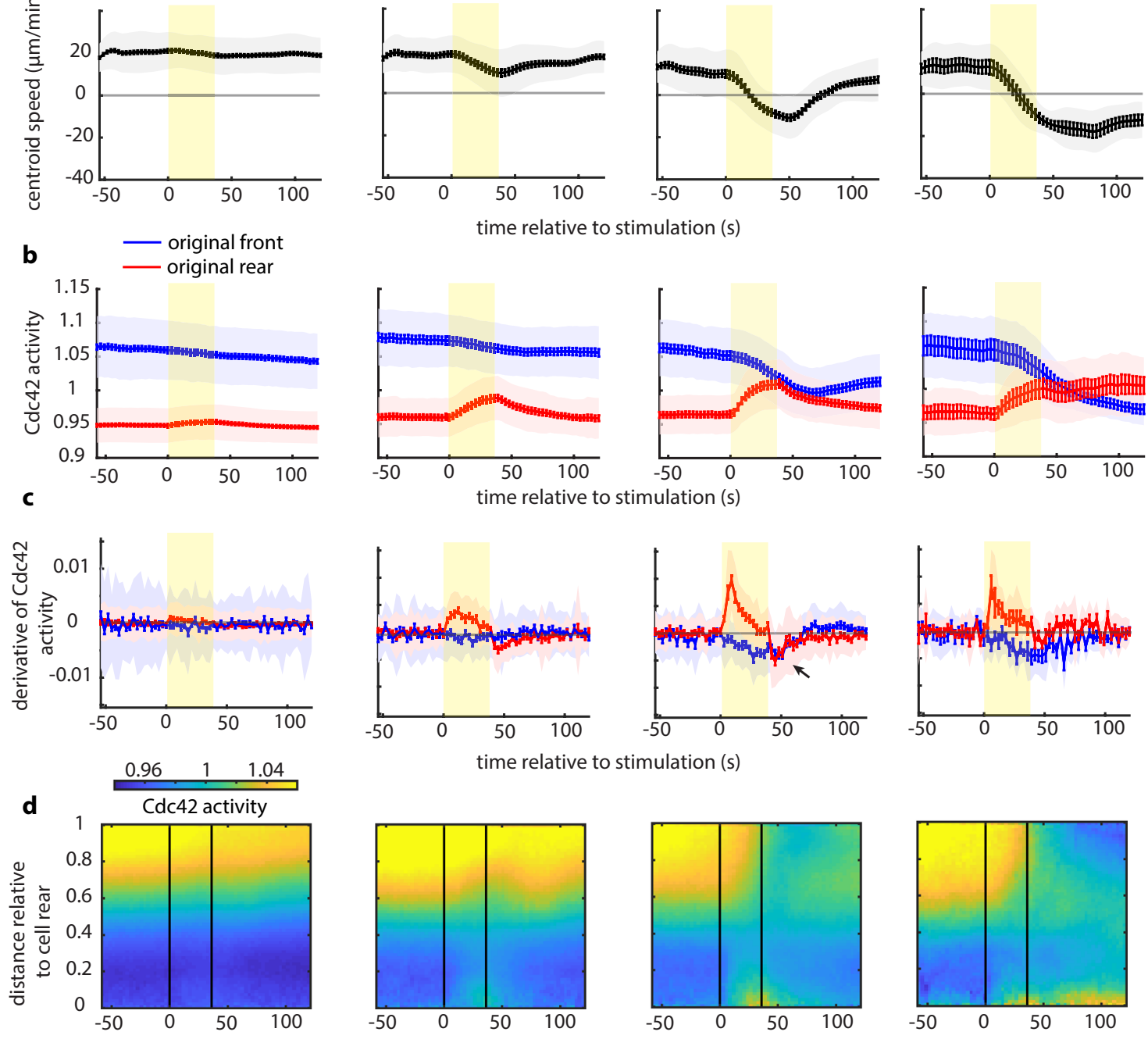

time relative to stimulation (s)

Fig. 4 A strong cell rear is refractory to receptor inputs. a-c Cell centroid speed (a), mean Cdc42 activity (b), and derivative of Cdc42 activity (c) at the original front (blue) and rear (red) over time for each cellular response (lines: means, shaded regions: SD, error bars: mean value \pm SE). Data are averages from $n=141$ non-responders, $n=49$ medium responders, $n=44$ strong responders, and $n=11$ reversers from 20 independent experiments. Rectangular yellow shaded region marks the start and end of the 12-pulse stimulation. $\mathbf{d}$ Average kymograph representation of Cdc 42 activity as a function of time ( $x$ axis) and vertical position relative to the cell rear ( $y$-axis) for non-responding, medium responding, strong responding, and reversing cells. Vertical black lines indicate the start and end of the pulsated stimulation. Source data are provided as a Source Data file.

derivative of the Cdc42 activity at each edge (Fig. $4 b, c)$. The derivative revealed a key difference between the cells in the strong response and the reversed classes. The original rear of strong responding cells showed both a strong positive response to the initiation of stimulation and a prominent negative overshoot post stimulation (black arrow on the third panel of Fig. 4c). In contrast, reversers showed a lower positive response and no overshoot. Simply put, a cell that overreacted when the stimulation commenced would also overreact when it stopped, emerging as a strong responder. This combination of positive and negative regulation of $\mathrm{Cdc} 42$ downstream of receptors was also documented in cell-wide optogenetic stimulation experiments and may reflect temporal processing of input signals ${ }^{14}$.

Finally, average kymographs for the four distinct cellular responses compactly summarized on a whole-cell level the dynamic spatiotemporal response of Cdc42 activity in each cell class (Fig. 4d). The kymographs further highlighted our most surprising observation: that a strong rear can be refractory to receptor inputs, even at the level of signal transmission to Cdc42. In contrast, there was a clear trend in which a weaker rear can be amenable to receptor input and lead to a measurable whole-cell response. The migratory and signaling responses were qualitatively similar, regardless of whether a serum gradient was present (Fig. 4 and Supplementary Fig. 4). Overall, our observations reveal that the strength of the rear is key in modulating the magnitude of the behavioral response, raising the question of what tunes the rear's strength in the first place.

Perturbations that affect the phosphorylation of MRLC tune the amenability of the rear to respond to receptor inputs. We sought to explore what molecular pathways might be actively suppressing the rear response to receptor inputs. We considered two main candidates: (i) myosin II/RhoA and (ii) ERM (ezrin, radixin, and moesin) proteins/cortical actin. 
Myosin II and RhoA signaling have been extensively shown to limit membrane protrusion at the cell rear antagonizing front polarity signaling $3,15,16$. Myosin motor activity and myosin filament assembly are regulated by the phosphorylation of Ser19 and Thr18 on the MRLC ${ }^{17-19}$. Phosphorylation of MRLC in Dictyostelium discoideum has been shown to regulate stable polarity and the ability of the cell to form new lateral protrusions ${ }^{20}$. RhoA activates Rho-associated kinase (ROCK), which directly phosphorylates MRLC and inhibits the phosphatase PP1 with its regulatory myosin-binding subunit MYPT121 (Fig. 5a). ROCK inhibition with
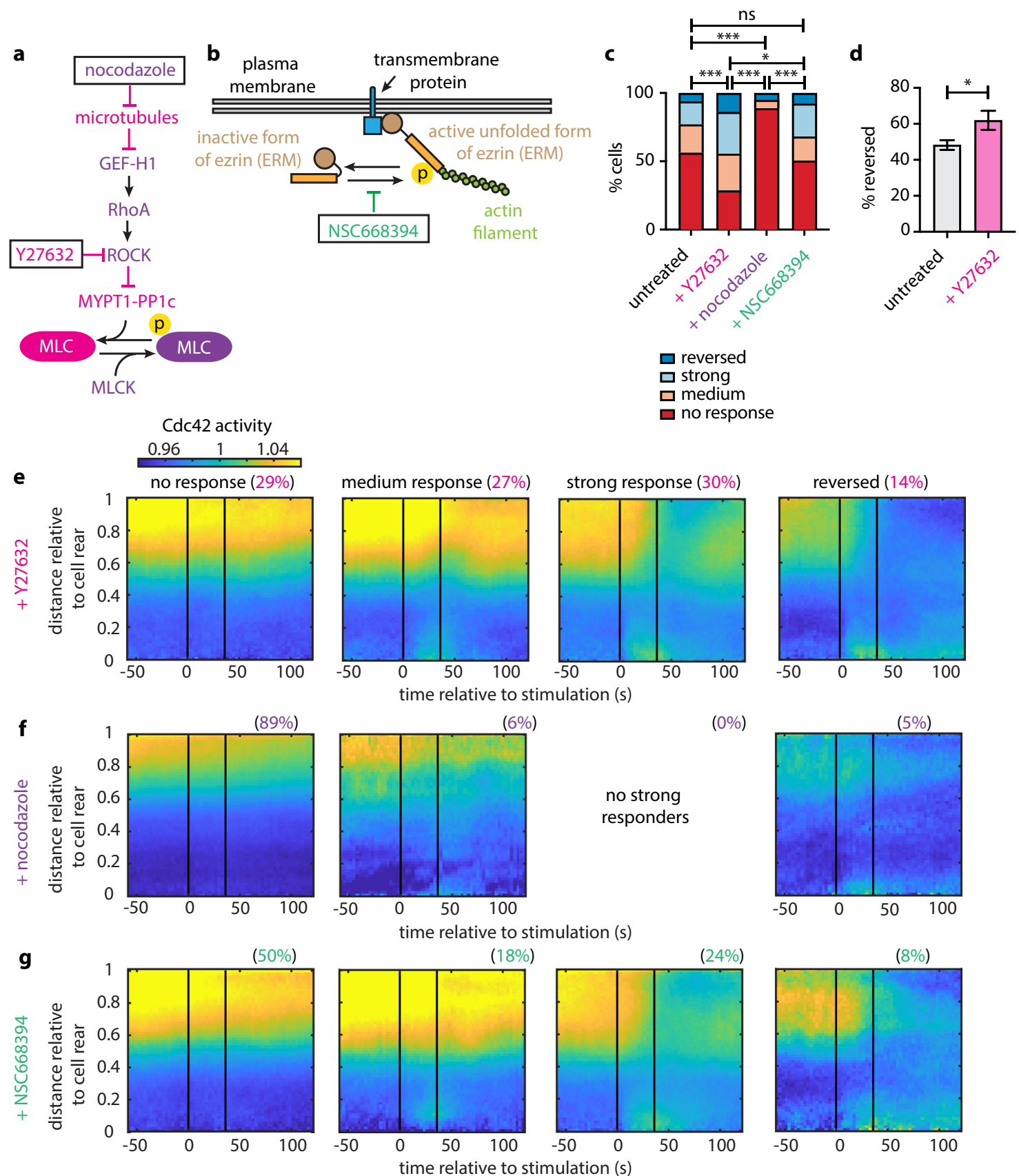

Fig. 5 Perturbations that affect the phosphorylation of myosin regulatory light chain tune the amenability of the rear to respond to receptor inputs. a Diagram of the signaling cascade regulating MRLC phosphorylation. A protein or perturbation that increases or decreases MRLC phosphorylation is colored with purple or magenta, respectively. b Sketch showing how the ERM proteins establish crosslinks between the plasma membrane and cortical actin. c Stacked bar plots of the percentage of cells exposed to transient stimulation that showed no response, medium response, strong response, and reversed for $n=261$ untreated cells, for $n=115$ Y27632-treated cells, $n=99$ nocodazole-treated cells, and $n=91$ NSC668394-treated cells (from 20, 7, 7, and 6 independent experiments, respectively); $p$-values of two-sided Fisher's exact tests ( ${ }^{\star} p<0.05,{ }^{\star \star *} p<0.001$, ns: $\left.p>0.05\right)$. $\mathbf{d}$ Bar plot of the percentage of Cdc42-expressing cells that reversed in response to persistent stimulation for $n=336$ untreated cells and $n=84$ Y27632-treated cells (from 36 and 6 independent experiments, respectively). Error bars represent confidence intervals assuming a binomial distribution around the cumulative mean of each group. Two-sided Fisher's exact test revealed a significant difference between the two conditions $\left({ }^{\star} p<0.05\right)$. e-g Average kymograph representation of Cdc42 activity as a function of time ( $x$-axis) and vertical position relative to the cell rear ( $y$-axis) for $n=107$ Y27632-treated cells (e), $n=76$ nocodazoletreated cells (f), and $n=84$ NSC668394-treated cells $(\mathbf{g})$. Vertical black lines indicate the start and end of the pulsated stimulation. Source data are provided as a Source Data file, including the exact $p$-values. 
Y27632 decreases MRLC phosphorylation in neutrophils ${ }^{22,23}$. Moreover, microtubules have been demonstrated to regulate MRLC phosphorylation by sequestering GEF-H1, a guanine nucleotide exchange factor of RhoA, in various cell types ${ }^{24-27}$. In neutrophils, microtubule destabilization results in activation of RhoA, hyperphosphorylation of MRLC, and enhanced myosin contractility 23,28 . Consequently, if the amenability of the cell rear to respond to receptor inputs is modulated by myosin II/RhoA activity, we would expect that Y27632 treatment would reduce the antagonistic effect of myosin II/RhoA and make the rear more amenable to optogenetic reprogramming. Conversely, nocodazole treatment, shown to globally increase RhoA activity and MRLC phosphorylation $23,29,30$, would be expected to further enhance the strength of the back polarity module, thus suppressing cell rear response.

ERM proteins have also been implicated in cytoskeletal remodeling and cell migration ${ }^{31,32}$. ERM proteins transition between an inactive/closed configuration in the cytosol and an active/phosphorylated form that links cortical actin to the plasma membrane $^{33}$ (Fig. 5b). Evidence suggests that the ERM protein moesin in its active/phosphorylated form is a key factor in neutrophil polarization inhibiting the small GTPases Rac, Rho, and Cdc42, as well as protrusion at the cell rear ${ }^{34}$. In addition, the persistence of directional migration of neutrophil-like cells towards AMLF gradients has been proposed to depend, at least in part, on the polarization of ERM proteins and moesin towards the cell rear ${ }^{12}$. Specifically, treatment of neutrophils with NSC668394, a quinoline that inhibits ezrin phosphorylation at Thr567 and ezrin-actin binding, has been shown to result in a considerable reduction of directional memory ${ }^{12}$. The above observations made NSC668394 a key perturbation to assess the involvement of ERM proteins in creating a refractory cell rear. Overall, we found that NSC668394 had no significant effect, whereas Y27632 and nocodozole had highly significant and opposite effects (Fig. 5c), suggesting that the dominant effect on the rear sensitivity is through the myosin II/RhoA pathway rather than through ERM proteins.

Specifically, treating cells with Y27632 reduced the number of cells in the non-responding class (Fig. 5c), consistent with what we expected from our hypothesis that myosin II/RhoA could modulate the responsiveness of the rear. Interestingly, the nonresponders decreased in number by almost twofold as compared to control, the medium responders increased marginally (1.3fold), and the strong responders and reversers increased by 1.8 fold and 2.3-fold, respectively. The strong and the reversed classes gained more than what would be expected from just depleting the non-responders, suggesting that the Y27632 treatment pushes each cell along the response distribution rather than simply suppressing one particular class. Thus, Y27632 is likely not only making the rear more amenable to optogenetic inputs but also may act on a whole-cell backness component. Consistent with these results, Y27632 treatment also increased the percentage of reversing cells in response to persistent stimulation (Fig. 5d).

In contrast, nocodazole treatment resulted in a striking 1.6-fold increase of the no response class as compared to control (Fig. $5 \mathrm{c}$ ). The fraction of medium responding cells decreased by 3.6 -fold and no cells under this condition exhibited a strong response, whereas the fraction of cells that reversed was comparable to that in the control case. Nocodazole's effect of increasing the fraction of non-responders was exactly what we expected as the converse of the Y27632 finding, further strengthening the evidence for a role for the regulatory pathway upstream of MRLC phosphorylation in tuning the responsiveness of the rear to receptor inputs.

In addition, average kymograph representation of the Cdc42 activity for the tested perturbations showed a consistent trend: nonresponding cells were strongly polarized, whereas cells that showed some kind of response were progressively (from medium, to strong, to reversed) more and more weakly polarized (Fig. 5e-g).

Myosin II suppresses cellular reorientation and redistributes more slowly than the Cdc42 activity response. Overall, our results pointed to a model in which modulation of MRLC phosphorylation regulates the rear response to receptor inputs. To visualize myosin dynamics in optogenetic-driven reversals, we generated an HL60 cell line stably expressing fluorescently tagged MRLC (Myl9-mScarlet) and parapinopsin. We found that the myosin line exhibited a higher percentage of non-responding cells as compared to the Cdc42 line (Fig. 6a). Using flow cytometry, we confirmed that all cells expressed the parapinopsin construct (Supplementary Fig. 5a). This observation led us to hypothesize that the overexpression of MRLC might itself suppress cellular response, consistent with a causative role for myosin II activity in creating a refractory rear.

Myosin was polarized to the rear of cells in each response class, but with varying degrees of polarization (Fig. 6b and Supplementary Fig. 5b, also Supplementary Movie 4). The average centroid speed traces for the four classes (Fig. 6c) were qualitatively identical and temporally close to the ones computed from the Cdc42 line (Fig. 4a), allowing us to draw direct comparisons between Cdc42 activity and myosin dynamics.

We quantified the subcellular normalized myosin II localization for cells in each response class and found that myosin relocalization lags behind $\mathrm{Cdc} 42$ activity response (Fig. 6b), suggesting that Cdc42 activity is a faster representation of polarity change. Furthermore, the derivative of the normalized myosin at each edge reveals the same key difference we observed on a Cdc42 activity level between the strong and the reversed response (black arrow on the third panel of Fig. 5e). That is, strong responding cells "overreact" both at the onset and the end of stimulation compared to reversing cells.

Finally, average kymographs of intracellular myosin II further illustrated how myosin overexpression seemed to bias cell behavior, with non-reversing cells having higher myosin levels (Supplementary Fig. 5c). This trend was also captured by comparing the averaged pre-stimulation myosin signal over the entire cell body (Supplementary Fig. 5d). Importantly, the difference was not just in the overall myosin expression level but also in its subcellular distribution, reflecting stronger asymmetry in myosin activity. We quantified the pre-existing subcellular myosin localization (Supplementary Fig. 5e-g) and found a larger accumulation of myosin at the rear of nonresponding cells (Supplementary Fig. 5e). Overexpression may result in increased contractile activity and steeper myosin polarization, rendering the cell rear more refractory to receptor inputs.

Perturbations affecting the phosphorylation of MRLC alter the intracellular localization of myosin II and modulate the receptiveness of the rear to respond. Treating the myosin cell line with Y27632 and nocodazole revealed the same trends as noted before with the Cdc42 line: Y27632 significantly reduced the percentage of non-responding cells as compared to control, whereas nocodazole greatly increased it (Fig. 7a). Y27632 treatment significantly increased the percentage of reversing cells both in response to 12-pulse (Fig. 7a) and persistent stimulation (Supplementary Fig. 6a). Notably, the proportion of myosinexpressing non-responding cells was higher in all conditions as compared to the Cdc42-expressing cells under the respective treatment conditions, consistent with the hypothesis that the myosin line is less amenable to input simply due to overexpression of the fluorescently tagged MRLC. 
a

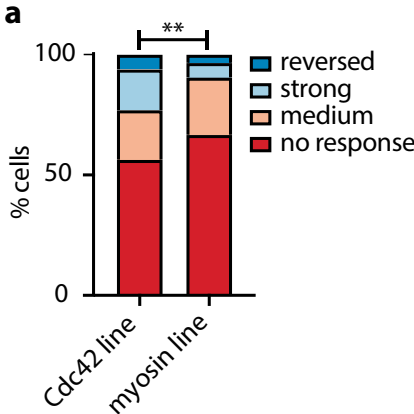

C
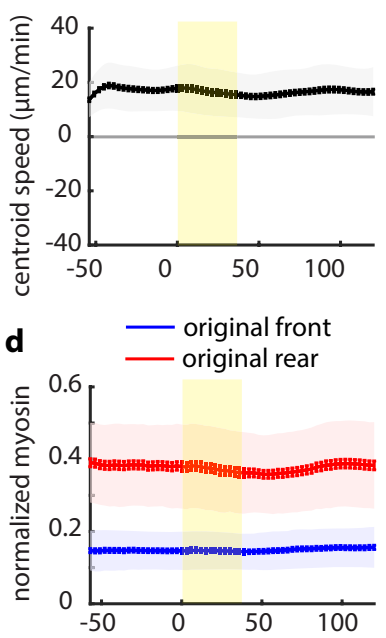

e

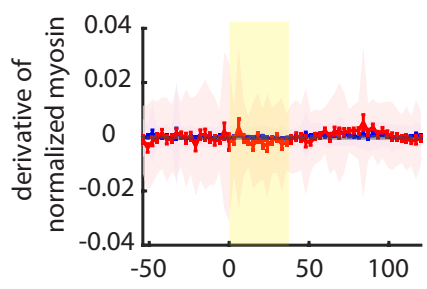

b

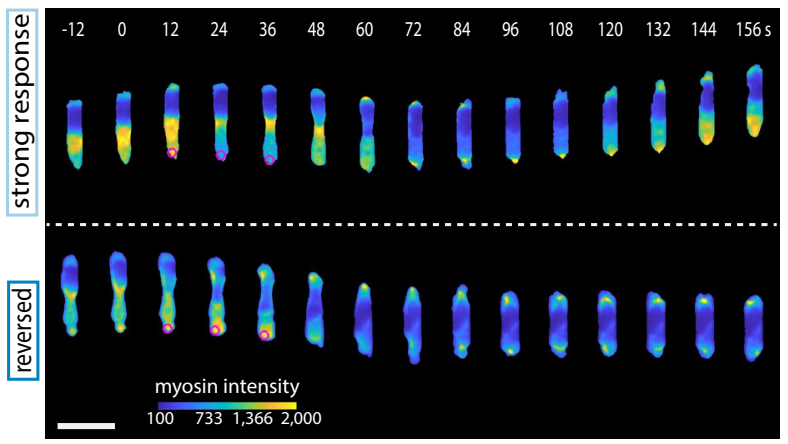

medium response (24\%)

strong response (6\%)

reversed (3\%)
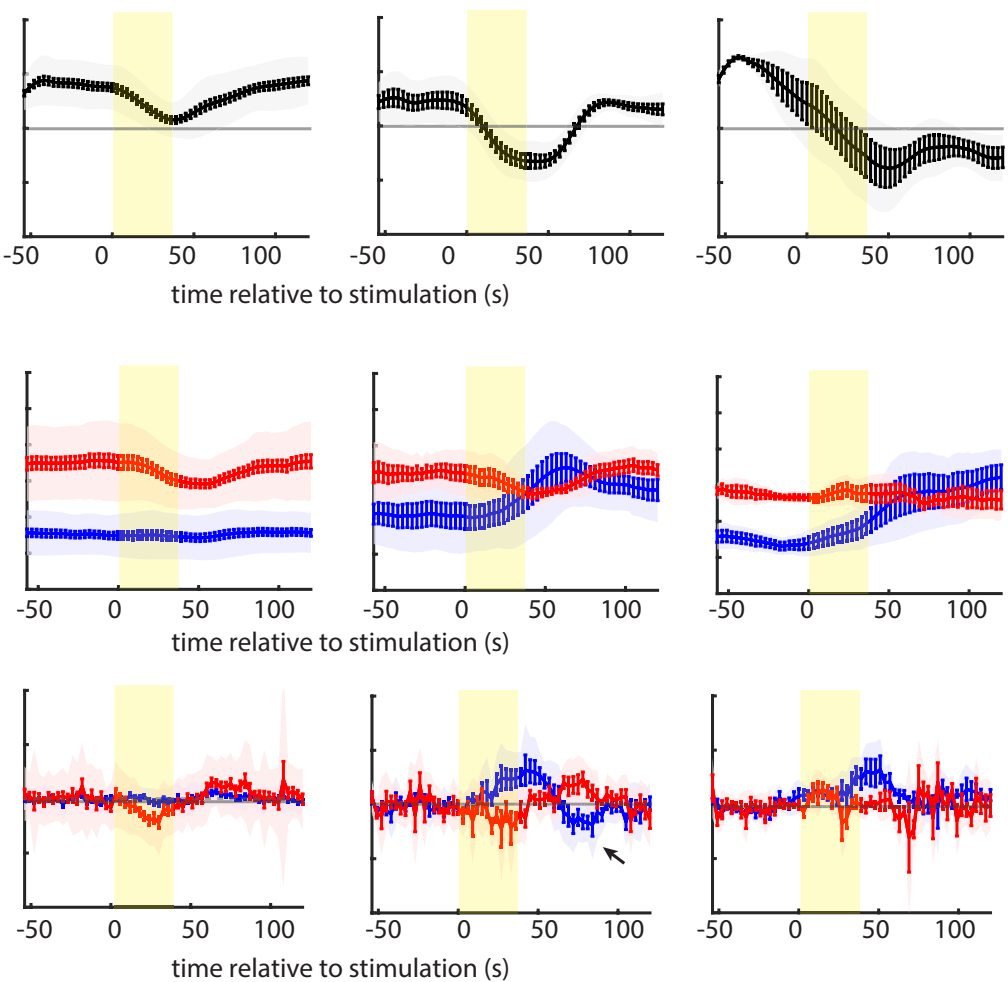

Fig. 6 Myosin II suppresses cellular reorientation and lags behind Cdc42 activity response. a Stacked bar plots of the percentage of cells exposed to transient stimulation that showed no response, medium response, strong response, and reversed for $n=261$ cells of the Cdc 42 line and $n=147$ cells of the myosin line (from 20 and 8 independent experiments, respectively); $p$-value of two-sided Fisher's exact test $\left({ }^{\star \star} p<0.01\right.$, exact $p$-value is provided in Source Data file). b Representative live-cell imaging snapshots of stimulation experiments with cells expressing parapinopsin and a myosin light chain sensor/ cytosolic tag, showing a strong response (upper panel) and a reversed response (lower panel). Cells migrated unperturbed for $60 \mathrm{~s}$ prior to starting a transient 12-pulse stimulation at their cell rear (magenta circles). Myosin intensity pseudo-colored to facilitate visualization. Images were captured every $3 \mathrm{~s}$ and subsampled for illustration purposes. Scale bar: $25 \mu \mathrm{m}$. c-e Mean cell centroid speed (c), normalized magnitude (d), and normalized derivative (e) of myosin intensity at the original front (blue) and rear (red) over time for each cellular response (lines: means, shaded regions: SD, error bars: mean value \pm SE). Data are averages from $n=98$ non-responders, $n=35$ medium responders, $n=9$ strong responders, and $n=5$ reversers. Rectangular yellow shaded region represents the start and stop of the pulsated stimulation. Source data are provided as a Source Data file.

We confirmed that drug perturbations were not affecting myosin expression (Supplementary Fig. 6b). However, the perturbations had measurable effects on subcellular myosin distribution, as quantified by front/rear myosin ratio (Fig. 7b), normalized front (Fig. 7c), rear (Fig. 7d) and middle myosin (Supplementary Fig. 6c), as well as cellular speed (Supplementary Fig. 6d). Y27632 greatly suppressed rear myosin as compared to the control and increased the normalized front myosin, resulting in a more symmetric myosin distribution. Acting opposite, nocodazole dramatically increased rear myosin and suppressed the front myosin pool. Average kymographs of myosin II for Y27632- and nocodazole-treated cells (Fig. 7e, f, respectively), and kymographs of the difference between each drug treatment and the control confirm the above (Supplementary Fig. 6e, f). In other words, nocodazole amplifies the myosin distribution asymmetry, whereas Y27632 eliminates it to a great extent. Notably, even upon Y27632 treatment 43\% of tagged MRLC-expressing cells were not responsive, suggesting that ROCK-regulated myosin activity and asymmetry, while being a strong predictive component, is not exclusively regulating the rear responsiveness.

\section{Discussion}

In this study, we demonstrated a causative role for myosin II and RhoA activity in creating a stabilized cell rear that is refractory to 

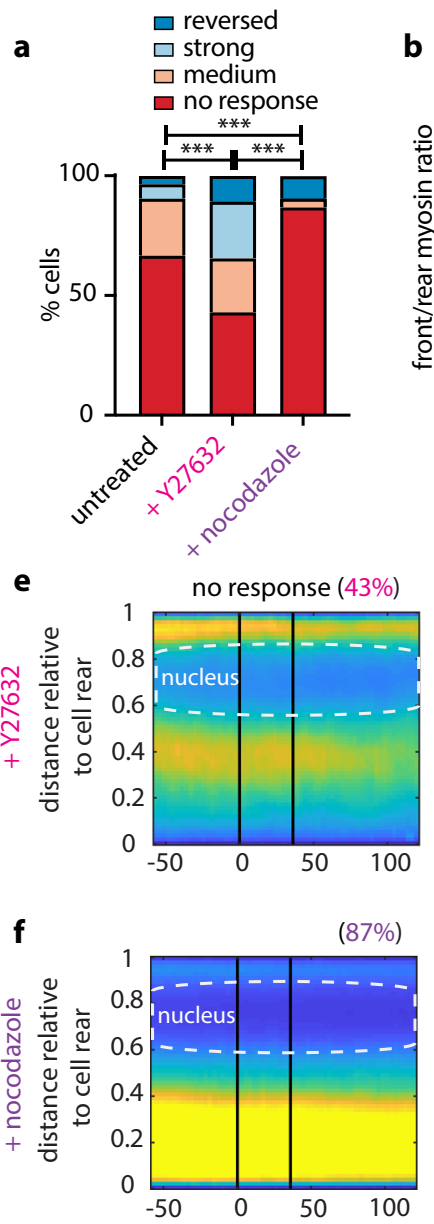
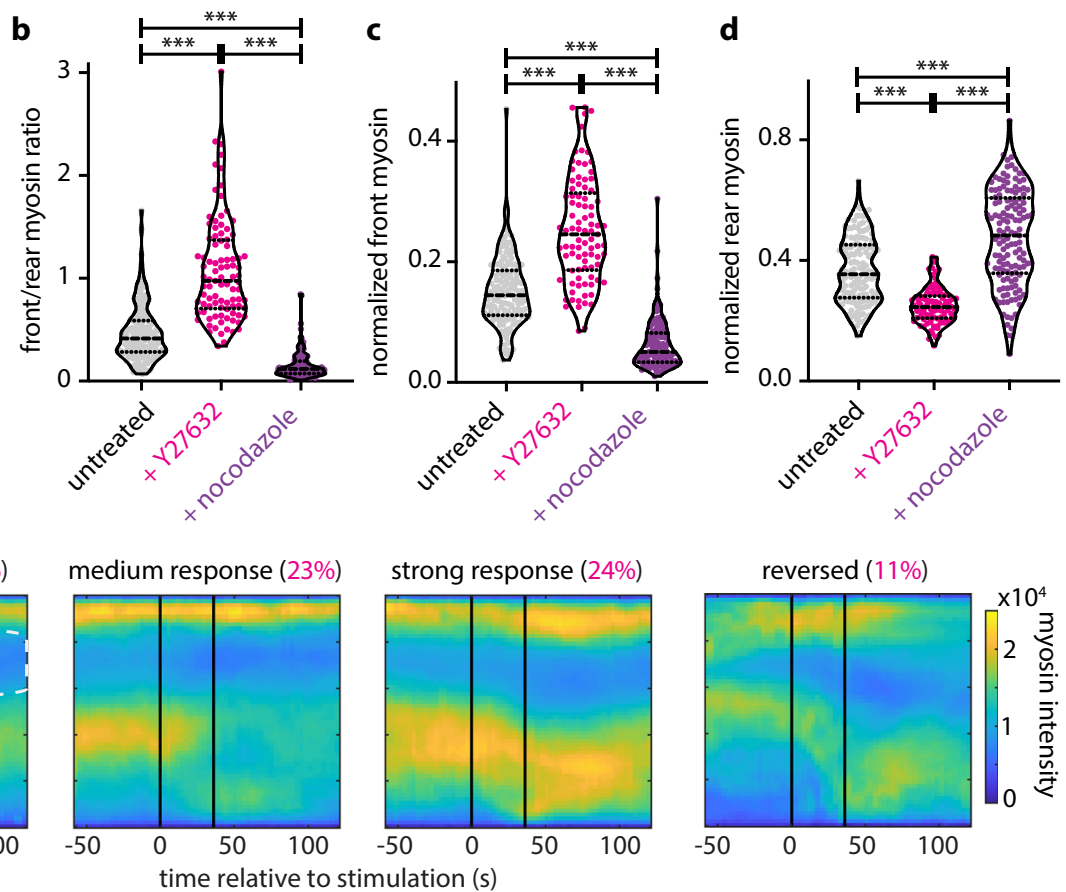

$(4 \%)$

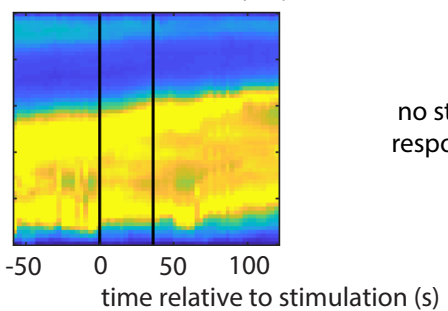

$(0 \%)$

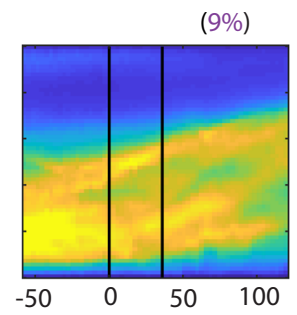

Fig. 7 Perturbations affecting myosin phosphorylation state alter the intracellular localization of myosin II and tune the sensitivity of the cell rear. a Stacked bar plots of the percentage of cells exposed to transient stimulation that showed no response, medium response, strong response, and reversed for $n=147$ untreated cells, for $n=93$ Y27632-treated, and $n=137$ nocodazole-treated cells (from 8, 10, and 8 independent experiments, respectively); $p$ values of two-sided Fisher's exact tests $\left({ }^{\star \star *} p<0.001\right)$. b-d Violin plots of mean front/rear myosin ratio (b), normalized front myosin (c), and normalized rear myosin (d) averaging over $15 \mathrm{~s}$ prior to stimulation for the same conditions and cells shown in $\mathbf{a}$; $p$-values of two-sided Wilcoxon's rank-sum test $\left({ }^{\star \star \star} p<0.001\right)$. e, f Average kymograph representation of myosin intensity as a function of time ( $x$-axis) and vertical position relative to the cell rear ( $y$ axis) for cells treated with Y27632 $(n=93)(\mathbf{e})$ and nocodazole $(n=137)(\mathbf{f})$. Vertical black lines indicate the start and stop of the pulsated stimulation. Source data are provided as a Source Data file, including exact $p$-values.

receptor inputs. We showed that the asymmetric localization of myosin II, being a readout of myosin activity, correlates with the strength of the rear, and that increased myosin II in the cell rear suppresses responsiveness to receptor inputs. Simply put, the higher the myosin levels and the greater the asymmetry, the more likely cells are to be non-responsive. Furthermore, we found that Y27632 and nocodazole perturbations, which are known to have opposite effects on the RhoA/ROCK/myosin II pathway (through decreasing and increasing the phosphorylation of MRLC, respectively), tune the rear's ability to respond to new receptor inputs and reorient migrating neutrophils.

Our results extend a longstanding model that antagonism between "frontness" and "backness" activities stabilizes polarity and causes asymmetric responses to receptor inputs. However, it had been difficult to distinguish whether this asymmetry was due to feedback-based competition between front and back programs or interference with signal transmission from receptors. Our approach allowed us to surgically examine the latter hypothesis by activating receptors only at the rear of cells without perturbing the cell front in a constrained environment. Rather than simply limiting the ability of new inputs to develop a fully functional front and reverse the cell, we found that a high level of myosin II activity at the cell rear was capable of blocking any detectable response downstream of receptors at the level of Cdc42 activity, in many cells.

For cells that maintain persistent polarity, such as neutrophils, there are several models describing how long-range communication underlying directional decisions might occur. A longstanding model in the field, abbreviated as LEGI (local-excitation-global-inhibition), suggests that, in addition to generating local protrusive signals, receptor activation also generates a chemical long-range global inhibitor to suppress protrusion at distant sites. The sequence of repolarization events described in Fig. 2 reveals that the stimulated cell rear quickly assumed front character and the long-distance effects did not appear to be transmitted to the previous cell front until the cell was physically stretched. Our results are consistent with a simple mechanical coupling between the two cell edges 8 , without the need to invoke an additional LEGI-type chemical signal.

In addition, through transient optogenetic stimulation, we discovered a particularly interesting class of cellular response, where cells transiently changed direction of motion and 
repolarized their Cdc42 activity and myosin but reverted back to their original polarity after stimulation ceased. This strong response is reminiscent of a previously documented cellular behavior in which a subset of neutrophil-like cells retained their directional memory after the removal and later reintroduction of an external fMLF chemotactic environment ${ }^{12}$. This directional memory was in part associated with ERM protein moesin, as inhibition through NSC668394 reduced this behavior. In our assay, treatment with NSC668394 did not result in a similar suppression of strong responding cells. This leads us to believe that the strong response in our assay is primarily resulting from the sensitivity to signal onset and removal we previously described. We note that nocodazole treatment resulted in no strong responding cells, which may suggest that microtubules or RhoA confer some biochemical memory of the original polarity. Decoupling a role for microtubules from RhoA is particularly complicated because of the known effect of microtubules to regulate MRLC phosphorylation by sequestering GEF-H1, such that microtubule destabilization triggers activation of RhoA, enhancing myosin II contractility ${ }^{23,28}$. However, nocodazole treatment also resulted in a dramatic increase in the fraction of non-responding cells, making it difficult to distinguish effects on biochemical memory from the general suppression of rear responsiveness.

Finally, by directly measuring Cdc42 activity in optogeneticdriven reversals of cells migrating inside $1 \mathrm{D}$ channels, we discovered that the direction of motion flipped before the polarity of Cdc42 activity flipped. In other words, it is not always the cell edge with more Cdc42 activity that defines the migratory direction. Our analysis may reflect neutrophils also engaging temporal-sensing mechanisms, evaluating how Cdc42 activity dynamically changes at each edge, rather than just comparing absolute or relative magnitudes of activity to dictate cytoskeletal polarization and ultimately migratory direction. A subtle point here is that although Cdc42 is a primary regulator of cell direction and formation of protrusive fronts in neutrophil-like cells ${ }^{4}$, there may be other components that could change faster. Nevertheless, evidence of temporal signal processing has also been shown in neutrophil-like cells when confined to migrate in a $2 \mathrm{D}$ plane under agarose ${ }^{14}$, in primary neutrophils upon fast switching of chemoattractant gradients ${ }^{35}$, and in myeloid cells whose persistent migration to certain intermediate chemokines involves temporal sensing ${ }^{36}$.

Historically, the cytoskeleton has usually been perceived as an effector operating downstream of signaling due to receptor inputs. However, growing evidence highlights that the system can also work the opposite way around, with the cytoskeleton critically influencing receptor activity ${ }^{37}$. Our findings strongly support this more complex view (Fig. 8), revealing a new dimension of the interplay between the cytoskeleton and signal transduction.

\footnotetext{
Methods

Generation of HL60 cell lines stably expressing parapinopsin-Cdc42 FRET sensor and parapinopsin-Myl9. Zebrafish parapinopsina and Cdc42 Tom/Kat FRET sensor plasmids were cloned as described in the accompanying article by Bell et al. ${ }^{14}$. HL60 cell lines were generated using the second-generation lentiviral system to stably insert the parapinopsina gene. Next, the Cdc42 FRET sensor was stably integrated using the piggybac transposon system ${ }^{38}$. The sensor plasmid was co-electroporated at a 1:1 ratio with the piggybac transposase plasmid using the Neon electroporation transfection system (Thermo Fisher Scientific). We used pulse voltage $1350 \mathrm{~V}$ and pulse width $35 \mathrm{~ms}$.

The myosin light chain plasmid was generated using multi-part Gibson cloning where iRFP670 fluorescent protein (to enable cytosolic segmentation), a tandem P2AT2A element, and mScarlet-I fused to Myl9 were inserted into a lentiviral vector. The myosin construct was then stably inserted using the lentiviral system. HL60 cells expressing the myosin construct were selected using $10 \mu \mathrm{g} / \mathrm{ml}$ blasticidin (Corning 30-100-RB). The line was sorted for cells highly expressing the myosin light chain (mScarlet-I) and cytoplasmic iRFP670 using a Beckman Coulter Astrios
} EQ at the UC Davis Flow Cytometry Core Facility.
Sensing

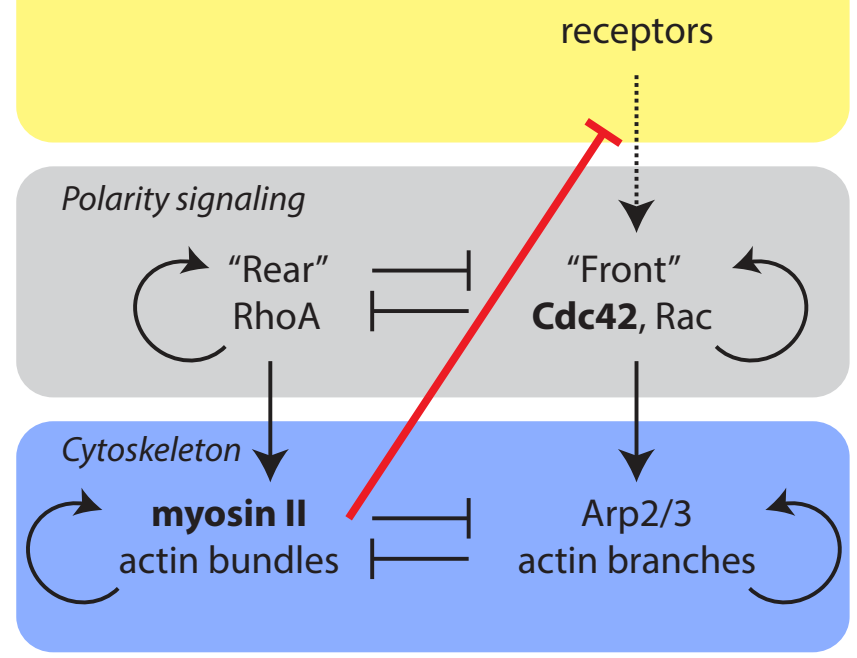

Fig. 8 Simplified model of cell polarization. Neutrophil polarization is thought to arise through antagonism between front and rear signaling modules. Information is typically thought to flow from a sensing module containing receptors (yellow layer) to the polarity signaling circuit (gray layer), which guides organization of the cytoskeleton (blue layer). At the front of the cell, Cdc42 and Rac lead to Arp2/3 actin branch formation, whereas RhoA regulates myosin II actin bundles at the cell rear (vertical arrows). The front and rear signaling modules are mutually exclusive ${ }^{3}$ and are governed by positive feedback loops (semicircular arrows) for selfamplification and polarity maintenance ${ }^{5-7}$. This particular signaling scheme has been demonstrated to be the most robust general motif that gives a stable polarization ${ }^{42}$. Similarly, the cytoskeleton also exhibits positive selfreinforcement and mutual inhibition by the different actin

organizations $23,43-46$. Signaling has usually been perceived to be upstream of the cytoskeleton, informing cytoskeletal responses. Our work revealed that myosin II is capable of blocking any detectable response downstream of receptor activation, even at the level of Cdc42 activity (red line).

The plasmids and plasmid maps are available on Addgene with IDs: 172472 (parapinospina), 172473 (Cdc42 Tom/Kat sensor), and 172474 (myosin light chain). All primers used in this study are listed in Supplementary Data 1.

Cell culture and differentiation. The HL60 cell line was a generous gift from Orion Weiner's lab. HL60 cells were cultured in RPMI 1640 plus L-glutamine and 25 mM HEPES media (Gibco RPMI 1640 Medium, HEPES, Thermo Fisher Scientific), supplemented with $10 \%$ heat-inactivated fetal bovine serum (hiFBS) (Foundation Fetal Bovine Serum, Gemini Bio, heated in a water bath for $40 \mathrm{~min}$ at $56^{\circ} \mathrm{C}$ to inactivate), $100 \mathrm{U} / \mathrm{mL}$ penicillin, $10 \mathrm{mg} / \mathrm{mL}$ streptomycin, and $0.25 \mathrm{mg} / \mathrm{mL}$ Amphotericin B (Gibco Antibiotic-Antimycotic, Thermo Fisher Scientific) ${ }^{39}$. Cells were maintained at $37^{\circ} \mathrm{C}$ and $5 \% \mathrm{CO}_{2}$ in a tissue culture incubator and were passaged every day so as to maintain a cell density close to $5 \times 10^{5}$ cells $/ \mathrm{mL}$. Cell differentiation into a neutrophil-like state was achieved by diluting cells in the complete RPMI media at a starting density of $2 \times 10^{5}$ cells $/ \mathrm{mL}$ and spiking $1.3 \%$ dimethylsulfoxide (DMSO) (Acros 61097). Experiments were performed with cells differentiated for 6 or 7 days.

Flow cytometry measurements of mCitrine-tagged parapinopsin expression. We used flow cytometry to measure the mCitrine-tagged parapinopsin receptor in the Cdc42- and myosin II-expressing HL60 cell lines. Roughly, $5 \times 10^{5}$ cells were centrifuged at $200 \times g$ for $5 \mathrm{~min}$ and resuspended in ice-cold fluorescence-activated cell sorting buffer (phosphate-buffered saline $+5 \%$ FBS $+0.01 \%$ sodium azide) Next, cells were analyzed using a Becton-Dickinson (BD) FACSCanto II Flow Cytometer. Cells were gated using a polygonal forward scatter (FSC) vs. side scatter (SSC) gate, to gate for viable cells. The gate was drawn manually to best separate the distinct two populations (Supplementary Fig. 1a). Data were collected using BD FACSDiva (Version 6.1.3) and analyzed using custom scripts in MATLAB. A threshold for "positive" presence of mCitrine was manually set at $10^{3}$ to separate the two peaks in a clearly bimodal distribution across samples (Supplementary Figs. $1 \mathrm{~b}$ and $5 \mathrm{a})$. 
Microfluidic device fabrication. Microfluidic devices consisted of a cell-loading channel of $200 \mu \mathrm{m}$ height and $200 \mu \mathrm{m}$ width bordered on one side by an array of straight, $944 \mu \mathrm{m}$-long migratory channels of $6 \mu \mathrm{m}$ width and $3 \mu \mathrm{m}$ height. Migration channels were arrayed in groups of three, each triplet connecting to a common $4 \mathrm{~nL}$ volume reservoir.

Devices were fabricated using photolithography and soft-lithography approaches ${ }^{40}$. Briefly, photolithography masks were designed using AutoCAD software (Autodesk) and provided in chrome on glass by Front Range Photomask (Lake Havasu City, AZ). The master wafer was prepared by sequentially spincoating the wafer with two layers of SU-8-negative photoresist (Microchem, Newton, MA) and patterning by exposure to ultraviolet (UV) light through the two chrome masks. Two cycles were carried out to produce the migration channels at $3 \mu \mathrm{m}$ height and the loading channels and reservoirs at $200 \mu \mathrm{m}$ height. To fabricate polydimethylsiloxane (PDMS) devices, the master wafer was used as a replica mold for soft lithography. PDMS (Fisher Scientific, Fair Lawn, NJ) was mixed thoroughly with a curing agent at a ratio of $10: 1$, poured over the master wafer, then degassed for $1 \mathrm{~h}$ prior to curing in an oven at $75^{\circ} \mathrm{C}$ overnight. Once cooled, PDMS devices were cut from the master wafer and the inlets and outlets punched using a $1 \mathrm{~mm}$ biopsy punch (Harris Uni-Core, Ted Pella, Inc., Redding, CA). Following treatment with oxygen plasma, devices were then bonded irreversibly to $35 \mathrm{~mm}$ glass-bottom (No. 0 coverslip) $\gamma$-irradiated culture dishes (MatTek, Corp., Ashland, MA) by heating to $85^{\circ} \mathrm{C}$ for $10 \mathrm{~min}$ on a hotplate.

Microfluidic device priming. Similar to previously published ${ }^{41}$, to prime the device $20 \mu \mathrm{L}$ of L-15 media (Gibco) containing $20 \%$ hiFBS was pipetted through one of the two loading ports. Ten microliters of the same solution was pipetted on top of each loading port. The device was then placed in a vacuum desiccator connected to house vacuum (27 in $\mathrm{Hg}$ ) for $10 \mathrm{~min}$. Upon removal, the device was rested for an additional $10 \mathrm{~min}$, until the attractant filled entirely the straight migration channels, connected orthogonally to the central loading channel. The device was then washed twice, by pipetting into a loading port $200 \mu \mathrm{L}$ of L-15 media. These washing steps removed the attractant from the central loading channel and its passive diffusion from the migration channels into the central loading channel established a serum gradient. To prevent evaporation, $3 \mathrm{~mL}$ of L-15 media were added to the glass-bottom dish to cover the device.

Retinal preparation. Preparation of retinal stock solutions was performed in a dark room with red-light sources ${ }^{14}$. In brief, 9-cis-Retinal (Sigma Aldrich R5754) was dissolved in 200 proof ethanol (Sigma Aldrich) purged with argon gas, resulting in a concentration of $10 \mathrm{mg} / \mathrm{mL}$. Aliquots were stored at $-80^{\circ} \mathrm{C}$ in amber glass tubes (Sigma Aldrich).

$0.1 \mathrm{~g}$ of bovine serum albumin (BSA), Fraction V-Low-Endotoxin Grade (Gemini Bio 700-102 P), was vigorously mixed in $10 \mathrm{~mL}$ of L-15 media (Gibco), to prepare a $1 \%$ BSA solution. In darkness, $10 \mu \mathrm{L}$ of retinal stock was diluted to a working concentration of $10 \mu \mathrm{g} / \mathrm{mL}$ by gradually adding the $1 \%$ BSA solution $(9 \times 10 \mu \mathrm{L}, 9 \times 100 \mu \mathrm{L}, 2 \times 500 \mu \mathrm{L}, 8 \times 1 \mathrm{~mL})$ until all $10 \mathrm{~mL}$ were used. The final retinal solution was kept in darkness, on a rocker located in a standard cold room, and was left to incubate overnight. Final retinal solutions were used for experiments within 2 days after preparation.

Retinal incubation and cell loading. Differentiated HL60 cells $\left(3 \times 10^{5}\right)$ were spun down at $200 \times g$ for $5 \mathrm{~min}$ and resuspended in $1 \mathrm{~mL}$ of retinal solution to incubate at $37^{\circ} \mathrm{C}$ for $1 \mathrm{~h}$. This incubation and all remaining cell handling happened in darkness with red-light sources. Incubated cells were spun down at $200 \times g$ for $10 \mathrm{~min}$ and were resuspended in $\sim 20 \mu \mathrm{L}$ of $\mathrm{L}-15$. Out of this cell suspension, $10 \mu \mathrm{L}$ were pipetted into the loading port of the device. The device was then transferred on the microscope at $37^{\circ} \mathrm{C}$, where the cells would be imaged.

Pharmacological perturbations. To explore the role of myosin II/RhoA and ERM proteins/cortical actin in creating a cell rear refractory to receptor inputs, we pretreated cells with either $20 \mu \mathrm{M} \Upsilon$-27632 (Sigma) or $50 \mu \mathrm{M}$ nocodazole (Sigma), or $50 \mu \mathrm{M}$ NSC668394 (Sigma), for the last $30 \mathrm{~min}$ of retinal incubation. Cells were spun down at $200 \times g$ for $5 \mathrm{~min}$ and were resuspended in $\sim 20 \mu \mathrm{L}$ of L-15 containing the same final drug concentration. Of this cell suspension, $10 \mu \mathrm{L}$ were loaded in a device that was pretreated with the same drug concentration. The pharmacological compound was included in both the attractant solution and the L-15 solution used for washing, in the same final concentration. For all pharmacological perturbations, DMSO was used as a vehicle. As a control, we performed experiments with $0.25 \%$ DMSO to match the maximum final DMSO concentration in the drug treatments.

Fluorescence microscopy. Fluorescence microscopy was performed on a Ti-E inverted Nikon microscope with a XLED1 LED light source for epi-fluorescence illumination. Images were acquired every $3 \mathrm{~s}$ on two Andor Zyla $4.2 \mathrm{sCMOS}$ cameras, using a Cairn TwinCam LS image splitter equipped with a dichroic mirror (Chroma ZT594rdc, $\sim 605 \mathrm{~nm}$ edge wavelength) and emission filters to allow simultaneous imaging. The Cdc42 TomKat FRET imaging was performed as described in the accompanying article by Bell et al. ${ }^{14}$. For myosin-imaging experiments, we used a 405/488/561/640 nm quad band filter cube (Chroma 91032) that allowed rapid, sequential $407 \mathrm{~nm}$ optogenetic stimulation and simultaneous imaging of mScarlet-I-Myl9 ( $561 \mathrm{~nm}$ excitation) and cytoplasmic infrared fluorescent protein (iRFP) ( $\sim 40 \mathrm{~nm}$ excitation). Bandpass emission filters were used to eliminate bleedthrough of iRFP into the myosin channel, but some bleedthrough of mScarlet signal into the iRFP channel was unavoidable and tolerated to allow simultaneous imaging. Rapid and precise stage movements were achieved using an ASI stage (MS-2000 Flat-top) equipped with linear encoders. The microscope was controlled through custom-built MATLAB R2015a software (MathWorks) interfaced with Micro-Manager (Version 1.4.23) to automate cell stimulation and timelapse microscopy protocols, enabling highly reproducible experimental conditions. Cells were imaged with a $\times 60$ oil-immersion objective (Nikon Apochromat 1.49 NA) and were maintained at $37^{\circ} \mathrm{C}$ using a temperature and humidity control unit (OkoLab Microscope Lexan Enclosure). Each experiment was terminated within $2 \mathrm{~h}$ of imaging.

Stimulation assays. Cell stimulation and imaging was performed using a custommade MATLAB R2015a interface for Micro-Manager (Version 1.4.23). Subcellular opsin stimulation was performed using a $407 \mathrm{~nm}$ laser (Coherent Cube) with a custom fiber coupling inserted in a fluorescence recovery after photobleaching (FRAP) port on the microscope. Both the TomKat dichroic and the dichroic used for myosin imaging can pass $407 \mathrm{~nm}$ light, enabling rapid FRAP stimulation without the need to swap cubes. To focus the FRAP module, HL60 cells expressing either the TomKat FRET sensor or the mScarlet-I-Myl9 were loaded in a microfluidic device as described above. Cells were imaged, as described, to determine the appropriate focal plane. We selected cells, zapped them by setting the laser to $40 \mathrm{~ms}$ exposure and $10 \%$ power, and imaged them using the FRAP channel. The $x-y$ translation knobs of the FRAP module were adjusted to bring the FRAP spot near the center of the image (around 512 pixel $\times 512$ pixel on a 1024 pixel $\times 1024$ pixel image, where 1 pixel is $0.21 \mu \mathrm{m}$ ). Thereafter, we used the $z$-adjustment knob to focus the FRAP spot into a tight approximately Gaussian-shaped spot. We measured the power of the FRAP laser at the objective using a Thorlabs handheld optical power meter (PM100D) and a microscope slide power sensor (S170C). Cellstimulation experiments were conducted using $\sim 1.8 \mu \mathrm{W}$ power measured at the objective. On each experimental day, pictures of the FRAP spot were collected and averaged to identify the pixel with the maximum FRAP spot intensity. The $x-y$ coordinates of the maximum intensity pixel were saved and later used to dynamically translate the stage, so the maximum activation spot coincided with the desired subcellular stimulation location.

Each lane on the microfluidic chip was organized into a grid of $x-y$ coordinates that were imaged consequently. Cells were segmented in real time using a minimum fluorescence threshold, and minimum and maximum size thresholds. The target point was computed by determining the cell centroid (in the center stimulation assay) or the cell rear (in all other cases). Determination of the cell rear relied on extracting the cell body boundary from the binary mask and determining the point in the cell perimeter as specified by a target angle (for us $180^{\circ}$ in respect to the original orientation of cell movement). For persistent stimulation experiments, UV stimulation and imaging were alternated until the imaging period was over. For the 12-pulse stimulation assays we administered a total of 12 pulses of light, whereas for center stimulation assays we administered a total of 5 pulses. For all assays, we maintained the same time interval $(3 \mathrm{~s})$ for imaging and stimulation. In all assays, cells were allowed to migrate unstimulated for 21 frames and right after imaging the 21st frame, we commenced the subcellular stimulation, alternating between imaging and stimulation. This results in turning the receptor off with each image and then rapidly back on again with the following stimulation pulse, as parapinopsina is a $\mathrm{G}_{\alpha \mathrm{i}}$-family coupled GPCR that is activated by UV light and inactivated by orange light $(>530 \mathrm{~nm})^{14}$, leading to rapid activation and deactivation cycles. Thus, our stimulation assays are pulsed.

Camera and illumination corrections. Raw images were corrected for the camera dark-state noise, for differences in the camera chip sensitivity, and for dust in the light path as described in the accompanying article by Bell et al. ${ }^{14}$. Moreover, a gradient in apparent FRET ratio activity was empirically observed from the top to the bottom of the TomKat FRET sensor images due to imperfections in the light path. To correct for this gradient, we developed a ratio correction image. Images of unstimulated Cdc42 TomKat FRET sensor HL60 cells loaded in microfluidic channels were collected systematically with different stage positions and the same $\times 60$ objective so that at least one cell was imaged on every portion of the camera sensor. We computed FRET ratios using our standard analysis pipeline for each image and assembled the images into a 3D image stack. We took the median of the FRET ratio over the stack (including only pixels corresponding to cells) to generate a single representative full-field FRET image. To reduce local variability, we then smoothed this image by taking the median over each 24 pixel $\times 24$ pixel block and smoothing using a Gaussian filter $(\sigma=5)$. We then smoothly resized the result to generate a "ratio correction image" with the same dimensions as the input image. We applied the correction by dividing the FRET ratio images by the ratio correction image. We note that this last correction was not necessary for the Myl9 imaging.

Cell segmentation and image background subtraction. Raw FRET pair images were registered using the coordinate-mapping strategy described ${ }^{14}$. Cell 
segmentation was performed on the sum of the aligned FRET donor and acceptor images, to improve signal-to-noise ratio. First, we conservatively defined background and cell object pixels. A background image was then determined using the median intensity of background pixels in the local neighborhood of each pixel. The background image was subtracted from the sum image. Object edges were enhanced by first smoothing the image using a broader Gaussian filter $(\sigma=5)$ and then subtracting the smoothed image from the original image. Finally, the cell object binary masks were determined through the Otsu's threshold method. For each FRET donor and acceptor image, we subtracted the background as defined through the above segmentation strategy. Pixels not included in the cell mask were defined as not a number, so as to eliminate them from downstream analysis. The FRET ratio image was calculated as FRET acceptor divided by FRET donor. We used a similar strategy to register the mScarlet-I-Myl9 and cytoplasmic iRFP pair images for the myosin cell line and to subtract the background.

Movie processing. For the Supplementary Movies 1-3, each donor and acceptor image was smoothed using a Gaussian filter $(\sigma=2)$, after subtracting the background as described above. We note that this smoothing step was only applied to movies and not for any other analysis. The FRET ratio image was calculated, once again, as FRET acceptor divided by FRET donor (using the smoothed images). The same smoothing strategy was applied for the mScarlet-I-Myl9 signal (Supplementary Movie 4). The Cdc42 activity ratio for each cell was a bit different and, as the relative activity across each cell is most informative, we chose to show cells in Supplementary Movies 1-3 with a FRET range from the 1st percentile to the 99th percentile of the corrected FRET ratio images on a movie-by-movie basis. For Supplementary Movie 4, as differences in myosin expression levels had functional consequences, we displayed myosin-expressing cells with fixed bounds from 100 to 2000 .

\section{Analysis of the center stimulation assay and threshold assignment for} responding cells. We quantified the mean $\mathrm{Cdc42}$ activity over the entire cell body for 60 cells and found a clear increase in $\mathrm{Cdc} 42$ activity for most of the cells that started immediately after the initiation of stimulation, peaking at $15 \mathrm{~s}$, right after the last stimulation pulse (Supplementary Fig. 1d). Cdc42 activity returned back to the baseline $15 \mathrm{~s}$ later ( $30 \mathrm{~s}$ after the first light pulse). We went on to quantify the relative increase of Cdc42 activity (Supplementary Fig. 1d) by breaking the cellular response into four windows ("Control 1" $=[-60:-42] \mathrm{s}$, "Control 2" $=[-39:-21] \mathrm{s}$, "Control 3" $=[-18: 0] \mathrm{s}$, and a "Peak" window centered at the maximum Cdc42 activity found between 3 and $33 \mathrm{~s}$ with a spread $\pm 9 \mathrm{~s}$ around that, to match the window size with one of the three other windows). For each cell, we quantified the mean value in each of the four windows and then computed a "control" response (by dividing the respective means of "Control 2" by "Control 1") and a "peak response" (by dividing the respective means of "Peak" by "Control 3"). The control distribution is symmetric around the median 0.9993. Leveraging that, we expected half of the inherently non-responding cells in the peak response distribution to have a relative increase $<0.9993$. Using this statistical argument, we estimated that $93 \%$ of cells were responsive (close to the $97 \%$ of cells that expressed the opsin). Applying a more stringent threshold at 1.008 (which was exceeded in the control ratio by only $5 \%$ of cells) provided a lower bound estimate of $70 \%$ responding cells. In both estimated thresholds, the percentage of inherently responding cells was significantly higher than the $47 \%$ of cells that reversed upon repetitive stimulation, suggesting that the suppressed percentage of reversing cells was due to some other kind of variation among the cells.

Analysis of the Cdc42 activity profile. To quantify the pre-stimulus Cdc42 activity profiles (Fig. 1d), we stratified cells as reversers and non-reversers, based on their migratory speed. We assumed and verified that cells are in a steady state for the $15 \mathrm{~s}$ prior to stimulation, so we averaged over that time window to minimize noise in our measurements. Specifically, for each cell we considered the six frames prior to stimulation onset, corresponding to $15 \mathrm{~s}$. For each time point, we computed a 1D profile of the Cdc42 activity, centered at the cell centroid and interpolated the Cdc42 activity profile over a fixed number of points (the average cell length) to account for variabilities in cell length. Interpolation was carried out via fitting a cubic smooth spline with the smoothing parameter set at the default value of 0.5 (where 0 fits a straight line and 1 gives a total fit). We computed a mean profile over each cell averaging these six interpolated profiles. As a final step, we averaged across all cells in each of the two groups and computed mean profiles (solid lines), SDs (shaded regions), and SEMs (error bars).

\section{Subcellular localization of Cdc42 activity and myosin II. Cell front and rear} areas were defined as the 800 pixels closest to the cell front and rear edge. The 800 pixels enabled us to capture the entire penetration depth of the Cdc42 activity at the cell front. We note that the order of events (Fig. 2) was qualitatively the same for different penetration depths $(800,400$, and 200 pixels): the stimulated rear responded first, then the front, followed by the cell reversing its direction of migration, before the Cdc42 crossover point. To define the cell middle, we computed the major axis of the segmented cell body, divided it into three equidistant length segments, and took a rectangular window around the middle part of the cell. For each area of interest (front, rear, and middle), we computed the Cdc42 activity as the sum of the donor signal divided by the sum of the acceptor signal in that area. We used the same strategy to identify the front, rear, and middle of Myl9expressing cells, so that we can directly compare the dynamic relocalization of Cdc42 activity and myosin II (Fig. 6c-e). To analyze the relative subcellular distribution of myosin, we computed the normalized myosin at the front, rear, and middle as the sum of the Myl9 signal in each area of interest divided by the sum of the Myl9 signal in the entire segmented cell body (Figs. 6d, e and 7e, f, and Supplementary Figs. 5e-g and 6c).

Cell speed, length, and area analysis. Cell centroid speed was computed by dividing the displacement of the cell's centroid between two consecutive frames with the time interval between frames $(3 \mathrm{~s})$. For each cell, its speed trace was then smoothed with a local regression using weighted linear least squares and a seconddegree polynomial model ("loess" or locally weighted smoothing in MATLAB). We used a span of $10 \%$ of the total number of data points for this smoothing. We then computed the mean cell speed averaging these smoothed speed traces (Figs. 2c, g, 4a, and 6c, and Supplementary Fig. 4a).

Cell length was approximated via computing the major axis of the binary cell mask, whereas cell area was calculated by summing the total number of pixels of the computed binary cell mask. Relative changes in length and area were plotted by dividing the cell length and area for each cell by its respective length and area before stimulation (Fig. 2d).

Determination of the order of events. Temporal determination of the order of events relied on interpolating the time between the closest time points to key events, namely rear response, front response, cell stalling, and Cdc42 activity crosspoint. Stimulated rear response was defined as a $1 \%$ increase in the Cdc42 activity at the rear, whereas front response was defined as a $1 \%$ decrease in Cdc42 activity at the front from the steady-state baseline values for rear and front at $t=0 \mathrm{~s}$. Cell stalling was defined as the interpolated time point where the cell's centroid speed was zero and Cdc42 cross-point as the interpolated time point where the Cdc42 activities of front and rear intersected. This temporal analysis was performed both on average trends (Fig. 2a-c) and on a single-cell level (Fig. 2e, f).

Stratification into cellular responses. Cells were stratified into reversers and non-reversers based on whether they responded to the persistent optogenetic stimulation by reversing their direction of migration and Cdc 42 activity axis (Fig. 1c-g). Transient stimulation resulted in additional cellular responses, namely no response, medium response, strong response, and stably reversing cells (Figs. 3-5). We classified "no response" cells as cells that showed no or minimal rise $(<2 \%)$ in $\mathrm{Cdc} 42$ activity at their rear, when compared to the pre-stimulation rear activity (averaging over $15 \mathrm{~s}$ prior to the initiation of stimulation). Medium responding cells were cells that showed some increase of $\mathrm{Cdc} 42$ activity at their rear $(>2 \%)$ but no engagement of the front (difference between minimum front Cdc42 activity and maximum back Cdc42 activity $>0.03$ ), often resulting in cell body elongation. Strong responding cells were classified as cells that showed engagement of both the rear and the front, but not stably reversing (many of these cells transiently reversed direction but flipped back to their original direction after the stimulus ended). Reversed cells were defined as described above. To extend this analysis to the myosin cell line, the method had to be adjusted, given the lack of a Cdc42 signal reporter in that line (Figs. 6 and 7). Similar to before, strong responding and reversed Myl9-expressing cells were classified based on reversing their myosin polarity and direction of migration transiently or stably, respectively (for Y27632-treated cells, only migratory speed was considered). Non-responding and medium responding Myl9-expressing cells were classified using a combination of migratory speed, morphology, and manual curation.

Kymograph representation for Cdc42 activity and myosin. For each cell and each time point, a 1D profile of either Cdc42 activity or myosin was computed from cell front to rear. These 1D profiles were aligned according to the cell centroid. To account for the fact that the cell length was variable across cells and time points, we took these 1D profiles and aligned them to a fixed average cell length through interpolation. For each time point, we took the interpolated 1D profiles and computed an average profile from all cells. Interpolation was carried out as described above, via fitting a cubic smooth spline with the smoothing parameter set at the default value of 0.5 . Synthesizing the average profiles along time, we constructed kymograph representations (Figs. 4d, 5e, g, and 7e, f, and Supplementary Figs. $4 \mathrm{~d}$ and $5 \mathrm{c}$ ).

Statistical analysis. For categorical data, day-to-day and experiment-toexperiment variation was consistent with counting noise and the observed SDs were reasonably explained by the binomial model in each case. For quantitative signaling, speed, and localization data, cell-to-cell variability was the dominant source of variability. Given the limited and variable number of cells that could be analyzed in each experiment, we felt that individual cells represented the most relevant independent unit for statistical analysis. We were also careful to perform control experiments on each day to avoid potential bias. Based on these considerations, we pooled data from all independent experiments performed. We indicate the number of cells and the number of independent experiments in the 
figure legends. All measurements were taken using independent cells. Statistical parameters and significance are reported in the figures and the figure legends. Data are determined to be statistically significant when $p<0.05$ by either Wilcoxon's rank-sum test or Fisher's exact test ("ranksum" or "fishertest" in MATLAB, respectively). All statistical tests were two-sided.

To depict the percentage of reversing cells (Fig. 1c and Supplementary Fig. 2d, e) under persistent stimulation, we represented data with bar plots showing the cumulative mean value and an error bar, which represents the confidence interval assuming a binomial distribution around the cumulative mean, pooling data from all independent experiments.

For the statistical analysis of the subcellular localization of $\mathrm{Cdc} 42$ activity and myosin II, as well as the centroid speed, we used violin plots pooling all cells that passed our pre-processing standards of masking and tracking, from all independent experiments (Figs. 1e, f, $3 c$, and 7b-d, and Supplementary Figs. 1a-c, 3a-d, f-j, $4 \mathrm{~d}-\mathrm{g}$, and $5 \mathrm{a}-\mathrm{c})$. In each violin plot, the dashed line represents the median of the distribution and the dotted lines the $25 \%$ and $75 \%$ quartiles of the distribution. To compare among groups (e.g., reversing and non-reversing cells or among cellular responses or drug perturbations), we performed non-parametric two-sided Wilcoxon's rank-sum tests.

To depict the percentage of cells in each response class under titrated stimulation, we used stacked bar plots (Figs. 3b, 5c, 6a, and 7a, and Supplementary Fig. 3e). We performed the Fisher's exact test on the distributions as a whole using "fisher.test" in R that generalizes for tables larger than 2 by 2 , to examine whether at least one of the classes differed between compared pairs ( $p$-values are reported on the figures and the Source Data file). In addition, we computed the two-sided Fisher's exact test for each cellular response class between conditions (e.g., comparing the reversers in untreated cells vs. Y27632-treated cells, etc.) using "fishertest" in MATLAB, to probe which classes significantly changed. Based on those two considerations, we highlighted the significant differences in the main text.

Plotting. Plotting was performed using MATLAB R2018a (MATHWORKS) and GraphPad Prism 8.

Reporting summary. Further information on research design is available in the Nature Research Reporting Summary linked to this article.

\section{Data availability}

All processed data generated in this study are provided in the Supplementary Information and Source Data files. Due to the large size of the full data set, raw images are not included but are available from the corresponding authors upon reasonable request. Source data are provided with this paper.

\section{Code availability}

The code we developed for automating experiments and data analysis is available from the corresponding authors upon request.

Received: 18 June 2021; Accepted: 29 September 2021; Published online: 16 November 2021

\section{References}

1. Iijima, M., Huang, Y. E. \& Devreotes, P. Temporal and spatial regulation of chemotaxis. Dev. Cell 3, 469-478 (2002).

2. Servant, G. et al. Polarization of chemoattractant receptor signaling during neutrophil chemotaxis. Science 287, 1037-1040 (2000).

3. $\mathrm{Xu}, \mathrm{J}$. et al. Divergent signals and cytoskeletal assemblies regulate selforganizing polarity in neutrophils. Cell 114, 201-214 (2003).

4. Yang, H. W., Collins, S. R. \& Meyer, T. Locally excitable Cdc42 signals steer cells during chemotaxis. Nat. Cell Biol. 18, 191-201 (2016).

5. Hind, L. E., Vincent, W. J. B. \& Huttenlocher, A. Leading from the back: the role of the uropod in neutrophil polarization and migration. Dev. Cell 38, 161-169 (2016).

6. Wang, F. et al. Lipid products of $\mathrm{PI}(3) \mathrm{Ks}$ maintain persistent cell polarity and directed motility in neutrophils. Nat. Cell Biol. 4, 513-518 (2002).

7. Weiner, O. D. et al. A PtdInsP(3)- and Rho GTPase-mediated positive feedback loop regulates neutrophil polarity. Nat. Cell Biol. 4, 509-513 (2002).

8. Houk, A. R. et al. Membrane tension maintainscell polarity by confining signals to the leading edge during neutrophil migration. Cell 148, 175-188 (2012).

9. Zigmond, S. H., Levitsky, H. I. \& Kreel, B. J. Cell polarity: an examination of its behavioral expression and its consequences for polymorphonuclear leukocyte chemotaxis. J. Cell Biol. 89, 585-592 (1981).

10. Dehghani Zadeh, A., Seveau, S., Halbwachs-Mecarelli, L. \& Keller, H. U. Chemotactically-induced redistribution of CD43 as related to polarity and locomotion of human polymorphonuclear leucocytes. Biol. Cell 95, 265-273 (2003).

11. Gerisch, G. \& Keller, H. U. Chemotactic reorientation of granulocytes stimulated with micropipettes containing fMet-Leu-Phe. J. Cell Sci. 52, 1-10 (1981).

12. Prentice-Mott, H. V. et al. Directional memory arises from long-lived cytoskeletal asymmetries in polarized chemotactic cells. Proc. Natl Acad. Sci. USA 113, 1267-1272 (2016).

13. Skoge, M. et al. Cellular memory in eukaryotic chemotaxis. Proc. Natl Acad. Sci. USA 111, 14448-14453 (2014).

14. Bell, G. R. R., Rincón, E., Akdoğan, E. \& Collins, S. R. Optogenetic control of receptors reveals distinct roles for actin- and Cdc42-dependent negative signals in chemotactic signal processing. Nat. Commun. https://doi.org/ 10.1038/s41467-021-26371-z (2021).

15. Smith, L. A., Aranda-Espinoza, H., Haun, J. B., Dembo, M. \& Hammer, D. A. Neutrophil traction stresses are concentrated in the uropod during migration. Biophys. J. 92, L58-L60 (2007).

16. Uchida, K. S. K., Kitanishi-Yumura, T. \& Yumura, S. Myosin II contributes to the posterior contraction and the anterior extension during the retraction phase in migrating Dictyostelium cells. J. Cell Sci. 116, 51-60 (2003).

17. Craig, R., Smith, R. \& Kendrick-Jones, J. Light-chain phosphorylation controls the conformation of vertebrate non-muscle and smooth muscle myosin molecules. Nature 302, 436-439 (1983).

18. Smith, R. C. et al. Regulation of myosin filament assembly by light-chain phosphorylation. Philos. Trans. R. Soc. Lond. B. Biol. Sci. 302, 73-82 (1983).

19. Heissler, S. M. \& Sellers, J. R. Myosin light chains: teaching old dogs new tricks. BioArchitecture 4, 169-188 (2014).

20. Zhang, $\mathrm{H}$. et al. Phosphorylation of the myosin regulatory light chain plays a role in motility and polarity during Dictyostelium chemotaxis. J. Cell Sci. 115, 1733-1747 (2002).

21. Kimura, K. et al. Regulation of myosin phosphatase by rho and rho-associated kinase (rho-kinase). Science 273, 245-248 (1996).

22. Niggli, V. Rho-kinase in human neutrophils: a role in signalling for myosin light chain phosphorylation and cell migration. FEBS Lett. 445, 69-72 (1999).

23. Tsai, T. Y.-C. et al. Efficient front-rear coupling in neutrophil chemotaxis by dynamic myosin II localization. Dev. Cell 49, 189-205.e6 (2019).

24. Krendel, M., Zenke, F. T. \& Bokoch, G. M. Nucleotide exchange factor GEF$\mathrm{H} 1$ mediates cross-talk between microtubules and the actin cytoskeleton. Nat Cell Biol. 4, 294-301 (2002).

25. Matsuzawa, T., Kuwae, A., Yoshida, S., Sasakawa, C. \& Abe, A. Enteropathogenic Escherichia coli activates the RhoA signaling pathway via the stimulation of GEF-H1. EMBO J. 23, 3570-3582 (2004).

26. Birukova, A. A. et al. GEF-H1 is involved in agonist-induced human pulmonary endothelial barrier dysfunction. Am. J. Physiol. Lung Cell. Mol. Physiol. 290, L540-L548 (2006).

27. Chang, Y.-C., Nalbant, P., Birkenfeld, J., Chang, Z.-F. \& Bokoch, G. M. GEFH1 couples nocodazole-induced microtubule disassembly to cell contractility via RhoA. Mol. Biol. Cell 19, 2147-2153 (2008).

28. Niggli, V. Microtubule-disruption-induced and chemotactic-peptide-induced migration of human neutrophils: implications for differential sets of signalling pathways. J. Cell Sci. 116, 813-822 (2003).

29. Takesono, A., Heasman, S. J., Wojciak-Stothard, B., Garg, R. \& Ridley, A. J. Microtubules regulate migratory polarity through Rho/ROCK signaling in T cells. PLoS ONE 5, e8774 (2010).

30. Liu, B. P., Chrzanowska-Wodnicka, M. \& Burridge, K. Microtubule depolymerization induces stress fibers, focal adhesions, and DNA synthesis via the GTP-binding protein Rho. Cell Adhes. Commun. 5, 249-255 (1998).

31. Ivetic, A. \& Ridley, A. J. Ezrin/radixin/moesin proteins and Rho GTPase signalling in leucocytes. Immunology 112, 165-176 (2004).

32. Yoshinaga-Ohara, N., Takahashi, A., Uchiyama, T. \& Sasada, M. Spatiotemporal regulation of moesin phosphorylation and rear release by Rho and serine/threonine phosphatase during neutrophil migration. Exp. Cell Res. 278, 112-122 (2002).

33. Fehon, R. G., McClatchey, A. I. \& Bretscher, A. Organizing the cell cortex: the role of ERM proteins. Nat. Rev. Mol. Cell Biol. 11, 276-287 (2010).

34. Liu, X. et al. Moesin and myosin phosphatase confine neutrophil orientation in a chemotactic gradient. J. Exp. Med. 212, 267-280 (2015).

35. Irimia, D. et al. Microfluidic system for measuring neutrophil migratory responses to fast switches of chemical gradients. Lab. Chip 6, 191-198 (2006).

36. Petrie Aronin, C. E. et al. Migrating myeloid cells sense temporal dynamics of chemoattractant concentrations. Immunity 47, 862-874.e3 (2017).

37. Moujaber, O. \& Stochaj, U. The cytoskeleton as regulator of cell signaling pathways. Trends Biochem. Sci. 45, 96-107 (2020).

38. Yusa, K., Rad, R., Takeda, J. \& Bradley, A. Generation of transgene-free induced pluripotent mouse stem cells by the piggyBac transposon. Nat. Methods 6, 363-369 (2009).

39. Millius, A. \& Weiner, O. D. Chemotaxis in neutrophil-like HL-60 cells. Methods Mol. Biol. 571, 167-177 (2009). 
40. Boneschansker, L., Yan, J., Wong, E., Briscoe, D. M. \& Irimia, D. Microfluidic platform for the quantitative analysis of leukocyte migration signatures. Nat. Commun. 5, 4787 (2014).

41. Garner, R. M. et al. Neutrophil-like HL-60 cells expressing only GFP-tagged $\beta$ actin exhibit nearly normal motility. Cytoskeleton 77, 181-196 (2020).

42. Chau, A. H., Walter, J. M., Gerardin, J., Tang, C. \& Lim, W. A. Designing synthetic regulatory networks capable of self-organizing cell polarization. Cell 151, 320-332 (2012).

43. Bourne, H. R. \& Weiner, O. Cell polarity: a chemical compass. Nature 419 21-21 (2002).

44. Khaliullin, R. N. et al. A positive-feedback-based mechanism for constriction rate acceleration during cytokinesis in Caenorhabditis elegans. eLife 7, e36073 (2018).

45. Carlsson, A. E. Growth of branched actin networks against obstacles. Biophys. J. 81, 1907-1923 (2001).

46. Lomakin, A. J. et al. Competition of two distinct actin networks for actin defines a bistable switch for cell polarization. Nat. Cell Biol. 17, 1435-1445 (2015).

\section{Acknowledgements}

We thank Emel Akdogan for help with the flow cytometry measurements. We thank Tony Y.-C. Tsai and Effie Bastounis for insightful discussions and comments on the manuscript. We thank Bridgette McLaughlin at the UC Davis Cancer Center Flow Cytometry core (supported by P30 CA093373) for technical assistance with cell sorting. This work was supported by the Howard Hughes Medical Institute (J.A.T.) and an NIH Director's New Innovator Award (DP2 HD094656) to S.R.C. A.H. was a Stanford Bio-X Bowes Fellow, an Onassis Foundation Scholar, and was supported by the Foundation for Education and European Culture (IPEP), the A.G. Leventis Foundation, and the Attica Tradition Foundation. Microfabrication work was supported by NIG grant GM092804 to D.I. G.R.R.B. thanks the National Science Foundation Graduate Research Fellowship (Grant Number 1650042) for support.

\section{Author contributions}

A.H., G.R.R.B., S.R.C., and J.A.T. designed the experiments. A.H. performed the experiments and analyzed the data. A.H., G.R.R.B., and S.R.C. created software for data analysis and automating experiments. G.R.R.B. and S.R.C. developed the optogenetic and imaging tools. G.R.R.B. and S.S. created the cell lines. F.E. and D.I. designed the microfluidic devices. A.H., S.R.C., and J.A.T. interpreted the data. A.H., S.R.C., and J.A.T. wrote the manuscript. All authors read and approved the manuscript

\section{Competing interests}

The authors declare no competing interests.

\section{Additional information}

Supplementary information The online version contains supplementary material available at https://doi.org/10.1038/s41467-021-26622-z.

Correspondence and requests for materials should be addressed to Sean R. Collins or Julie A. Theriot.

Peer review information Nature Communications thanks the anonymous reviewer(s) for their contribution to the peer review of this work.

Reprints and permission information is available at http://www.nature.com/reprints

Publisher's note Springer Nature remains neutral with regard to jurisdictional claims in published maps and institutional affiliations.

Open Access This article is licensed under a Creative Commons Attribution 4.0 International License, which permits use, sharing,
adaptation, distribution and reproduction in any medium or format, as long as you give appropriate credit to the original author(s) and the source, provide a link to the Creative Commons license, and indicate if changes were made. The images or other third party material in this article are included in the article's Creative Commons license, unless indicated otherwise in a credit line to the material. If material is not included in the article's Creative Commons license and your intended use is not permitted by statutory regulation or exceeds the permitted use, you will need to obtain permission directly from the copyright holder. To view a copy of this license, visit http://creativecommons.org/ licenses/by/4.0/.

(C) The Author(s) 2021 